\title{
Characterization of Atomic and Electronic Structures of Electrochemically Active SOFC Cathode Surfaces
}

\author{
Final Report \\ Reporting Period Start Date: February 13, 2006 \\ Reporting Period End Date: August 12, 2009 \\ Principal Authors \\ Kevin Blinn, Yongman Choi, and Meilin Liu
}

Report Issued: November 9, 2009

DOE Contract No.: DE-FG26-06NT42735

Center for Innovative Fuel Cell and Battery Technologies

School of Materials Science and Engineering

Georgia Institute of Technology

Atlanta, GA 30332-0245 


\section{DISCLAIMER}

This report was prepared as an account of work sponsored by an agency of the United States Government. Neither the United States Government nor any agency thereof, nor any of their employees, makes any warranty, express of implied, or assumes any legal liability or responsibility for the accuracy, completeness, or usefulness of any information, apparatus, product, or process disclosed, or represents that its use would not infringe privately owned rights. Reference herein to any specific commercial product, process, or service by trade name, trademark, manufacturer, or otherwise does not necessarily constitute or imply its endorsement, recommendation, or favoring by the United States Government or any agency thereof. The views and opinions of authors expressed herein do not necessarily state or reflect those of the United States Government or any agency thereof. 


\begin{abstract}
The objective of this project is to gain a fundamental understanding of the oxygenreduction mechanism on mixed conducting cathode materials by means of quantumchemical calculations coupled with direct experimental measurements, such as vibrational spectroscopy.

We have made progress in the elucidation of the mechanisms of oxygen reduction of perovkite-type cathode materials for SOFCs using these quantum chemical calculations. We established computational framework for predicting properties such as oxygen diffusivity and reaction rate constants for adsorption, incorporation, and TPB reactions, and formulated predictions for LSM- and LSC-based cathode materials.

We have also further developed Raman spectroscopy as well as SERS as a characterization tool for SOFC cathode materials. Raman spectroscopy was used to detect chemical changes in the cathode from operation conditions, and SERS was used to probe for pertinent adsorbed species in oxygen reduction. However, much work on the subject of unraveling oxygen reduction for SOFC cathodes remains to be done.
\end{abstract}




\section{TABLE OF CONTENTS}

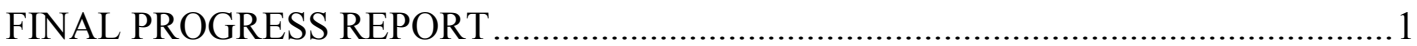

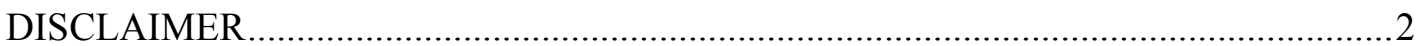

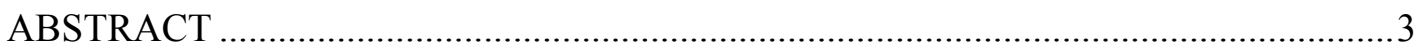

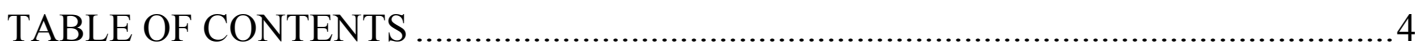

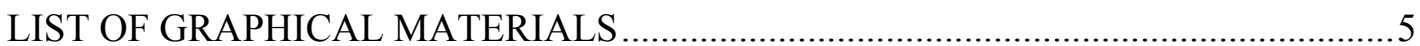

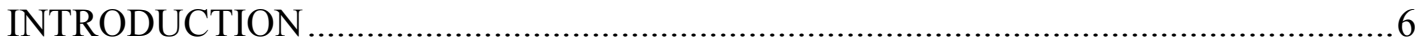

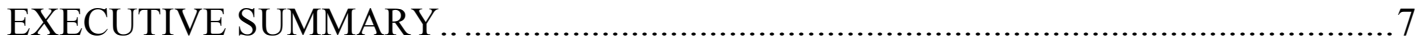

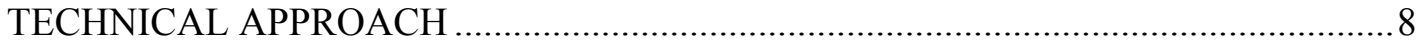

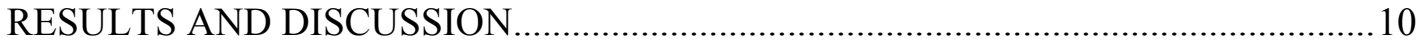

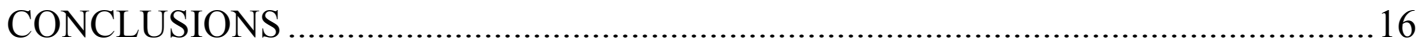

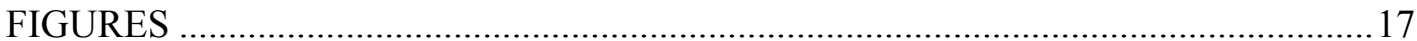

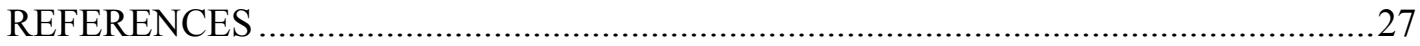




\section{LIST OF GRAPHICAL MATERIALS}

Figure 1 PES and geometrical structures of $\mathrm{O}_{2}(\mathrm{a}) \rightarrow 2 \mathrm{O}(\mathrm{a})$ dissociation processes on $\mathrm{Ag}$ covered Ceterminated $\mathrm{CeO}_{2}(111)$ surface. The black numbers are the relative energies referenced to $\mathrm{O}_{2}(\mathrm{~g}) /$ surfaces $(=0)$. The red numbers are the Bader charges of the adsorbed $\mathrm{O}$.

Figure 2 PES and geometrical structures of $\mathrm{O}_{2}(\mathrm{a}) \rightarrow 2 \mathrm{O}(\mathrm{a})$ dissociation processes on $\mathrm{Ag}(111)$ surface. The black numbers are the relative energies referenced to $\mathrm{O}_{2}(\mathrm{~g}) /$ surfaces $(=0)$. The red numbers are the Bader charges of the adsorbed $\mathrm{O}$.

Figure 3 The stepwise reaction mechanisms of oxygen reduction processes at $\mathrm{Ag}(111)$ and $\mathrm{Ag}(110)$ surfaces and at TPB, $\mathrm{CeO}_{2}(111)$-supported silver surface and Ce-terminated $\mathrm{CeO}_{2}(111)$ surface The underlined numbers represent the heat of reaction, $\Delta \mathrm{H}$, in $\mathrm{eV}$ whereas the italic numbers represent the reaction barrier, Ea, in $\mathrm{eV}$.

Figure 4 (a) Geometrical information of adsorbed oxygen species on a defective $\mathrm{LaMnO}_{3}$ surface. $\mathbf{V}$ and dashed circles denote an oxygen vacancy and adsorbed oxygen species on the surface, respectively. (b) Potential energy profiles at $0 \mathrm{~K}$ for the oxygen reduction reaction on a defective $\mathrm{LaMnO}_{3}$

surface.....

Figure 5 Molecular dynamics (MD) results simulated at $800^{\circ} \mathrm{C}$. $\mathbf{V}$ and dashed circles denote an oxygen vacancy and adsorbed oxygen species on the surface, respectively.

Figure 6 A stepwise reaction mechanism of oxygen reduction on a defective LMO surface.

Figure 7 A typical phase diagram for the interactions between $\mathrm{O}_{2}$ and $\mathrm{LaMnO}_{3}(100)$ and (110) surfaces at $1 / 6$ and $1 / 3$ coverage, respectively.

Figure 8 Potential energy profiles and top views of an intermediate state and products for $\mathrm{O}_{2-}$ $\mathrm{La}_{0.5} \mathrm{Sr}_{0.5} \mathrm{MnO}_{3-\delta}$ interactions. $\mathbf{V}$ denotes an oxygen vacancy. Dashed circles and $\mathbf{V}$ represent adsorbed oxygen species and an oxygen vacancy on the surface, respectively. Relative energies in brackets are ZPE-corrected.

Figure 9 Comparison of predicted rate constants for the dissociation process of $\mathrm{O}_{2}$ on the perfect $\mathrm{LaMnO}_{3}$ and $\mathrm{La}_{0.5} \mathrm{Sr}_{0.5} \mathrm{MnO}_{3-\delta}$. Solid lines are fitted using the linear-squared method...

Figure 10 . (a) Top views of the LSM50(100) surface model and stable states (O1 and O2) and a transition state of adsorbed oxygen species on LSM50. (b) Predicted surface diffusivities $\left(k_{\mathrm{SD}}\right)$ of adsorbed oxygen species on LSM50 and LSC50..

Figure 11 (a) SEM image of a dense LSM-GDC composite cathode fabricated by a co-pressing and co-sintering method. (b) Inverse polarization resistance versus TPB length. The values next to the data points are LSM volume percentages for the samples.

Figure 12 Raman spectra collected from various SSC samples modified with $20 \mathrm{~nm} \mathrm{Ag}$ colloid in air. Different SERS hotspots may enhance different species signals and thus different peaks.

Figure 13 Raman spectra for LSM and LSCF pellets in air after DC sputtering of Ag for 10 seconds...

Figure 14 Raman spectra collected at room temperature from LSCF with sputtered Ag under various controlled atmosphere conditions (argon and oxygen gases).

Figure 15 Raman spectra obtained from Ag colloid-treated LSCF surface at room temperature under controlled atmosphere.

Figure 16 (a) Raman spectrum collected from surface of LSCF film on GDC pellet in air before and after application of $0.8 \mathrm{~V} \mathrm{DC}$ polarization. A shift in the wavenumbers of the peaks can be seen, and the ratio of the larger peak to the smaller one also increases..(b) Raman spectra collected from powders of different LSCF ( $\left.\mathrm{La}_{1-\mathrm{x}} \mathrm{Sr}_{\mathrm{x}} \mathrm{Co}_{0.2} \mathrm{Fe}_{0.8} \mathrm{O}_{3-\delta}\right)$ compositions. The two marked peaks vary with change in $\mathrm{La} / \mathrm{Sr}$ ratio.

Figure 17 Raman spectra collected in air from sputter-deposited LSCF films on GDC substrates of various thicknesses, as well as a cylindrical pellet of LSCF. 


\section{INTRODUCTION}

The reduction processes of oxygen molecules comprise the key reaction in the cathode region of solid oxide fuel cells (SOFCs). Thus, unraveling the mechanisms of oxygen reduction and ionic conduction is a critical step toward the rational design of more efficient SOFC cathode materials. Because of the complexity of the involved charge and mass transfer processes, however, it is extremely difficult to probe the mechanistic details using the traditional experimental tools. For example, the oxygen reduction process may occur via several parallel reaction routes, depending on the ionic and electronic transport properties of the cathode and electrolyte materials $[1,2]$. In one example route, oxygen molecules are adsorbed on the three-phase boundary (TPB), where the electrolyte, electrode, and oxygen gas meet, and the reduced oxygen ions can directly combine with oxygen vacancies in the electrolyte. In another route, oxygen molecules are adsorbed and dissociated on the cathode surface, followed by the transport of the dissociated oxygen ions through the cathode to the electrolyte or along the surfaces of the cathode to the TPB. In a third route, oxygen molecules are adsorbed and/or dissociated on the electrolyte surface, followed by the transport of the adsorbed/dissociated oxygen ions along the surfaces of the electrolyte to the TPB, where they are reduced and incorporated into the electrolyte. The rate-limiting process and/or step within these routes remains unclear.

Thus, the main objective of the studies reported here is to gain such a fundamental understanding of the oxygen-reduction mechanism on mixed conducting cathode materials by means of quantum-chemical calculations coupled with direct experimental measurements, which mainly consist of normal and in-situ Raman spectroscopy of cathode material surfaces. Raman spectroscopy was chosen over other surface analysis methods due to its applicability towards in-situ experiments under fuel cell operating conditions. More conventional methods require vacuum environments to function.

The technical objectives include can be summarized as follows:

- Investigate $\mathrm{O}_{2}$ adsorption on simulated oxide-supported metal surfaces to serve as a model methodology for studying adsorption on cathode material surfaces

- Simulate the interactions of oxygen species and cathode materials under SOFC operating conditions (e.g., $\mathrm{T}=873$ to $1273 \mathrm{~K}$ at $\mathrm{pO}_{2}=0.2 \mathrm{~atm}$ ) by quantum molecular dynamics (QMD) and other computational methods.

- Elucidate the mechanisms for oxygen reduction on LSM-based materials and predict rate constants for individual steps by using quantum chemical calculations.

- Predict surface and bulk properties (e.g., diffusivities) of $\mathrm{La}_{0.5} \mathrm{Sr}_{0.5} \mathrm{MnO}_{3-\delta}$ and $\mathrm{La}_{0.5} \mathrm{Sr}_{0.5} \mathrm{CoO}_{3-\delta}$ cathode materials.

- Use vibrational spectroscopy to validate findings from the above by probing for oxygen species and directly investigate phenomena on the cathode surface, such as phase inhomogeneities and stoichiometric changes.

These objectives work towards obtaining information that can be used for more rational design of SOFC cathode materials in order to ultimately reduce SOFC technology and overall energy costs. 


\section{EXECUTIVE SUMMARY}

The objective of this project is to gain a fundamental understanding of the oxygenreduction mechanism on mixed conducting cathode materials by means of quantumchemical calculations coupled with direct experimental measurements using in-situ vibrational spectroscopy and impedance spectroscopy.

The approach begins with using an oxide-supported metal, and then $\mathrm{LaMnO}_{3}$ and $\mathrm{Sr}$ doped $\mathrm{LaMnO}_{3}$ in order to construct a surface model and methodology. Raman spectroscopy (ex-situ, in-situ, and surface-enhanced experiments) is then used to correlate theoretical models experimental conditions. In addition, Raman spectroscopy is explored as a means to characterize the cathode materials themselves in terms of phase inhomogeneities, stoichiometry changes, and cathode dimensions (i.e. film thickness), as all of these have a bearing on the oxygen reduction process. The correlation of structural calculations and spectroscopy results can help lead to an atomistic model for SOFC cathode performance.

\section{The main accomplishments can be briefly summarized as follows}

- Predicted the geometrical and electronic structures of the possible $\mathrm{O}_{2}$ adsorption as well as mechanisms of $\mathrm{O}_{2}$ reduction on the modeled surfaces of silver and $\mathrm{CeO}_{2}$ supported silver using DFT calculations and computed from potential energy surface (PES), respectively

- Showed that the oxygen reduction reaction on $\mathrm{LaMnO}_{3}$-based cathode materials may occur via a stepwise elementary reaction sequence

- Demonstrated through QMD simulations and statistical-theory techniques that defective $\mathrm{LaMnO}_{3}$ exhibits faster $\mathrm{O}_{2}$ dissociation kinetics than perfect $\mathrm{LaMnO}_{3}$, suggesting that oxygen vacancies play a critical role in oxygen reduction reactions

- Calculated diffusivity using transition state theory (TST) in order to find surface mobility and bulk oxygen ion conductivity for $\mathrm{LaCoO}_{3}$-based and $\mathrm{LaMnO}_{3}$-based cathode materials

- Found evidence of adsorbed and reduced oxygen species on cathode surfaces using SERS techniques

- Detected inhomogeneity and changes in stoichiometry on cathode material surfaces using Raman spectroscopy 


\section{TECHNICAL APPROACH}

To achieve the project objectives, we perform the following the following tasks:

\section{Task 1 - Quantum-chemical calculations}

Quantum-chemical calculations were performed, using methods such as quantum molecular dynamics (QMD), density functional theory (DFT), statistical-theory techniques, and transition-state theory to examine the reaction mechanisms for oxygen reduction on cathode materials. In this task, periodic slab models with vacuum spacer layers were used to construct more realistic surfaces and perform electronic structure calculations. For the electronic calculations, we employed standard approaches such as the VASP (Vienna ab initio simulation package) and Gaussaian03 software. We estimated the energetics of all possible intermediate species on the surfaces and propose feasible reaction pathways. We also predicted vibrational frequencies of the intermediates that were validated by Raman measurements performed in Task 2 . We also fabricated and characterized $\mathrm{La}_{0.85} \mathrm{Sr}_{0.15} \mathrm{MnO}_{3}(\mathrm{LSM})-\mathrm{Ce}_{0.9} \mathrm{Gd}_{0.1} \mathrm{O}_{1.95}$ (GDC) composite electrodes of different compositions to experimentally correlate three-phase boundary (TPB) length and interfacial polarization resistance and form a platform for characterization of surface catalytic properties of other electrode materials.

\section{Task 2 - In-situ vibrational spectroscopy studies of electrode materials}

Raman spectroscopy of the cathode materials under various conditions were used to follow the chemical evolution of reaction species at the electrode/gas interface. Normal Raman spectroscopy as well as SERS were used to probe for surface adsorbed species, structural inhomogeneities, and structural changes occurring in cathode materials as the operating conditions change. Conditions that were varied include temperature, applied voltage, and atmosphere. The effect of cathode film thickness on Raman spectra was also observed.

\section{The details of the technical approach are summarized as follows:}

\section{Calculations using oxide-supported metal surfaces}

The electronic calculation to identify the intermediate and transition states in the oxygen reduction process at the gas-phase interface (2PB) and three-phase boundary TPB are optimized by the spin-polarized density functional theory (DFT) with the projectoraugmented wave method (PAW) $[3,4]$. The exchange-correlation function is treated with the generalized gradient approximation (GGA) of PW91 formulation [5] for the total energy calculations. The Brillouin zone is sampled by Monkhorst-Pack scheme [6]. The 2PB is modeled by $\mathrm{Ag}(111)$ and $\mathrm{Ag}(110)$ surfaces, which are constructed by 6-layer (111) and (110) slabs. Both the (111) and (110) slabs have $2 \times 2$ surface cells with $4 \mathrm{Ag}$ atoms. The Monkhorst-Pack kpoints, applied for the super cells of $\operatorname{Ag}(111)$ and $\operatorname{Ag}(110)$ surfaces, are set as $8 \times 8 \times 1$ along $(11 \overline{2}) \times(1 \overline{1} 0) \times(111)$ and $8 \times 4 \times 1$ along $(1 \overline{1} 0) \times(001)$ $\mathrm{x}$ (110) directions, respectively. The cutoff energy and vacuum space are kept at $400 \mathrm{eV}$ and $10 \AA$, respectively. The top three layers are relaxed and the bottom three layers are fixed at the computed lattice constant during the structure optimization. The TPB is modeled by one $\mathrm{Ag}$ monolayer covered on $\mathrm{Ce}$ - or O-terminated $\mathrm{CeO}_{2}(111)$ surfaces. $\mathrm{CeO}_{2}(111)$ surface is constructed by a $\mathrm{p}(2 \times 2)$ 9-layer slab with $12\left[\mathrm{CeO}_{2}\right]$ units. The top three layers are relaxed, the Monkhorst-Pack k-points are set as $3 \times 3 \times 1$ along (211) x 
(011) x (111) directions, and a 10- $\AA$ vacuum space is presented along the (111) direction during the calculation.

\section{Calculations involving cathode material surfaces}

All periodic DFT calculations were performed using the Vienna ab initio simulation package (VASP). We applied the projector-augmented wave (PAW) method ${ }_{15}$ with a 400 $\mathrm{eV}$ cutoff energy to represent electron-ion interactions. The generalized gradient approximation with the Perdew-Wang (GGA-PW91) exchange-correlation functional was used. La, Sr, Mn, Co, and O atoms were described by $11\left(5 \mathrm{~s}^{2} 5 \mathrm{p}^{6} 5 \mathrm{~d}^{1} 6 \mathrm{~s}^{2}\right), 10\left(4 \mathrm{~s}^{2} 4 \mathrm{p}^{6} 5 \mathrm{~s}^{2}\right), 7$

$\left(4 s^{1} 3 d^{6}\right), 8\left(4 s^{1} 3 d^{7}\right)$ and $6\left(2 s^{2} s^{4}\right)$ valence electrons, respectively. Further details on these methodologies are well-documented elsewhere [7-10].

\section{Fabrication and characterization of LSM-GDC composite cathode}

A dense LSM-GDC composite electrode was fabricated with a GDC electrolyte layer to form a bi-layer structure using a co-pressing and co-firing process. LSM (Rhodia, average particle size: $1.1 \mu \mathrm{m}$ ) and GDC (Rhodia, average particle size: $0.3 \mu \mathrm{m}$ ) powders with various volume ratios ranging from 40:60 to 70:30 were mixed in a mortar further reducing particle sizes. The co-pressing consisted of several successive steps: the asprepared mixture powder was added to a die (diameter of $10 \mathrm{~mm}$ ) and tapped smooth using a pestle. Next, GDC powder was loaded into the die. The whole body was coldpressed under $200 \mathrm{MPa}$ into cylindrical pellets using a uniaxial die-press. After firing the samples at $1450{ }^{\circ} \mathrm{C}$ for $5 \mathrm{~h}$ in air, dense GDC/LSM-GDC wafers were attained. The thicknesses of GDC and LSM-GDC composite were $\sim 0.5 \mathrm{~mm}$ and $\sim 0.15 \mathrm{~mm}$, respectively. The composites were characterized by EDX, stereographic techniques for TPB length, and impedance spectroscopy for interfacial polarization resitance.

\section{Raman experiment details}

For SERS experiments, the candidate cathode materials SSC $\left(\mathrm{Sm}_{0.5} \mathrm{Sr}_{0.5} \mathrm{CoO}_{3-\delta}\right), \mathrm{LSM}$ $\left(\mathrm{La}_{0.85} \mathrm{Sr}_{0.15} \mathrm{O}_{3-\delta}\right)$, and LSCF $\left(\mathrm{La}_{1-\mathrm{x}} \mathrm{Sr}_{\mathrm{x}} \mathrm{Co}_{0.2} \mathrm{Fe}_{0.8} \mathrm{O}_{3-\delta}\right)$ were studied. Dense cathode surfaces were modified by nanoscale metal structures either through dropwise application of $20 \mathrm{~nm}$ colloidal silver or gold nanoparticles or DC sputtering of discontinuous silver thin films on surfaces. Samples for which the colloidal method was used were fired to $\sim 300^{\circ} \mathrm{C}$ in order to modify the structure of the surface nanoparticles. Surfaces were analyzed by Raman microscope either in ambient air or in a chamber capable of inducing different atmospheres and temperatures on the sample. Due to the tendency for favorable nanoscale metal structures on the surface to form "hot spots" where signal enhancement was highest but were not optically observable, spectra were collected from different spots in large rectangular meshes up to $100 \mu \mathrm{m}$ in length ranging from 500-1000 points. Some peaks in these spectra were assigned to possible adsorbed species. Additionally, conventional Raman spectroscopy was used to analyze LSCF before and after it was held under an intermediate SOFC operating temperature and high DC polarization. Its spectra were compared to powders anad targets of different stoichiometries to show changes in its surface chemistry following such treatment. In addition, cathode material thin films of different thicknesses deposited from targets fabricated from LSCF powders provided by Fuel Cell Materials were analyzed by Raman. These films were annealed at $800^{\circ} \mathrm{C}$ for 1 hour in 3 separate thermal cycles. LSCF thickness ranged from 80-200 nm. 


\section{RESULTS AND DISCUSSION}

\section{Oxide-supported metal surface calculations}

Calculations of adsorption energy suggest that the adsorptions on atop sites are less stable than those on 3-fold hollow sites, which can be rationalized from their bonding structures. Each adsorbed $\mathrm{O}_{2}$ at the atop position forms one chemical bond with single $\mathrm{Ag}$ atom; in contrast, the adsorbed $\mathrm{O}_{2}$ at the 3-fold hollow site forms more bonds with nearby $\mathrm{Ag}$ atoms. The calculations also suggest that the side-on adsorbed $\mathrm{O}_{2}{ }^{2-}$ (a) is more stable than the end-on adsorbed $\mathrm{O}_{2}^{-}$(a). In the end-on adsorption of superoxide only one $\mathrm{O}$ atom bonds with the surface while in side-on adsorption of peroxide both $\mathrm{O}$ atoms bond with the surface Ag atoms. The trends are consistent with the result of previous experimental observations $(9 ; 10)$. The analysis of the bond length indicate that, first, all adsorbed $\mathrm{O}_{2}(\mathrm{a})$ have longer bond lengths than that in the gas phase, $1.236 \AA$; second, the adsorbed $\mathrm{O}_{2}$ (a) with longer bond lengths has higher adsorption energies because the adsorbate can donate more electron and form stronger Ag-O bonds to stabilize the whole system. Therefore, the overall stability can be attributed to that the energy produced by forming strong $\mathrm{Ag}-\mathrm{O}$ bonds is more than the energy consumed by partially breaking the $\mathrm{O}-\mathrm{O}$ bond. The vibrational frequencies of the most stable superoxide and peroxide on the modeled TPB, atomic $\mathrm{Ag}$ covered on $\mathrm{Ce}$ - and $\mathrm{O}$-terminated $\mathrm{CeO}_{2}(111)$ surface, are computed. The adsorptions of superoxide forms with shorter $\mathrm{O}-\mathrm{O}$ bonds have higher vibrational frequencies $\left(1003-1079 \mathrm{~cm}^{-1}\right)$ than those of peroxide forms $\left(750-916 \mathrm{~cm}^{-1}\right)$. The $\mathrm{O}-\mathrm{O}$ vibrations on the modeled TPB are closer to the experimental observations of $\mathrm{O}_{2}$ (a) on the pure $\mathrm{CeO}_{2}$ (111) surface, $\mathrm{O}_{2}{ }^{-}$(a): $1127-1135 \mathrm{~cm}^{-1}$ and $\mathrm{O}_{2}{ }^{2-}$ (a): $831-877 \mathrm{~cm}^{-1}$ [11], than of $\mathrm{O}_{2}$ (a) on metal surfaces, $\mathrm{O}_{2}^{-}$(a): $870-1020 \mathrm{~cm}^{-1}$ and $\mathrm{O}_{2}{ }^{2-}$ (a): $610-660 \mathrm{~cm}^{-1}$ [12]. This implies that the $\mathrm{O}_{2}$ (a) on $\mathrm{Ag}$ covered $\mathrm{CeO}_{2}$ surface still retains the surface properties of $\mathrm{CeO}_{2}(111)$ rather than those of silver surfaces.

The reduction process is related to the $\mathrm{O}-\mathrm{O}$ dissociation process from the most stable $\mathrm{O}_{2}(\mathrm{a})$ adsorption. On the modeled TPB of $\mathrm{Ag}$ covered O-terminated $\mathrm{CeO}_{2}(111)$ surface, the transition state of $\mathrm{O}_{2}$ reduction process has a $0.43-\mathrm{eV}$ reaction barrier when $\mathrm{O}-\mathrm{O}$ bond is extended to $\sim 2 \AA$. The resulted product of the two dissociated $\mathrm{O}(\mathrm{a})$ prefers being adsorbed on the two nearest 3-fold hollow sites with an exothermicity of $-0.44 \mathrm{eV}$. On the other modeled TPB of $\mathrm{Ag}$ covered Ce-terminated $\mathrm{CeO}_{2}(111)$ surface, the $\mathrm{O}_{2}$ reduction process has a $0.28-\mathrm{eV}$ reaction barrier. The dissociated $\mathrm{O}$ atoms prefer bonding with subsurface $\mathrm{Ce}$ to surface $\mathrm{Ag}$ atoms with a much higher exothermicity of $4.70 \mathrm{eV}$ because the adsorption energies on atop sites of $\mathrm{O}-\mathrm{Ce}(5.55 \mathrm{eV})$ is much higher than that of $\mathrm{O}-\mathrm{Ag}(1.99 \mathrm{eV})$. The potential energy surface (PES) with Bader charge [13] analysis of this process is shown in Figure 1. As a result, the reduction processes on the two modeled TPBs have low reaction barriers and are highly exothermic. On the other hand, the reaction on the modeled $2 \mathrm{~PB}$ of $\mathrm{Ag}(111)$ and $\mathrm{Ag}(110)$ surfaces, the reduction processes have $0.85-\mathrm{eV}$ and $0.60-\mathrm{eV}$ reaction barriers, respectively. The PES of $\mathrm{O}_{2}$ dissociation process on $\mathrm{Ag}(111)$ surface with Bader charge analysis, for example, is shown in Figure 2. Unlike on TPB cases, the reduction processes on the modeled $2 \mathrm{~PB}$ are endothermic, $0.54 \mathrm{eV}$ on $\mathrm{Ag}(111)$ and $0.25 \mathrm{eV}$ on $\mathrm{Ag}(110)$ surfaces. Therefore, from the mechanism calculations, $\mathrm{O}_{2}$ reduction process is energetically more favorable to occur on TPB than $2 \mathrm{~PB}$ since the process has lower reaction barrier and are exothermic on TPB.

The $\mathrm{O}$ ion transport refers to the motion of the dissociated $\mathrm{O}(\mathrm{a})$ from one stable site to another on the surface. Comparing with surface morphology, the separation between the stable sites on TPB is larger than that on $2 \mathrm{~PB}$. Comparing with the electronic structure, the 
subsurface atoms of TPB has stronger interaction with the surface $\mathrm{O}(\mathrm{a})$ than $2 \mathrm{~PB}$.

Therefore, the transport process near TPB of Ag supported by O-terminated $\mathrm{CeO}_{2}(111)$ surface has higher energy barrier $(\sim 0.59 \mathrm{eV})$ than on $2 \mathrm{~PB}$ of silver: $\mathrm{Ag}(111)$ surface has a barrier of $0.37 \mathrm{eV}$ and $\mathrm{Ag}(110)$ surface of $0.17 \mathrm{eV}$. On the other modeled TPB of Ag supported by Ce-terminated $\mathrm{CeO}_{2}(111)$ surface, the $\mathrm{O}$ ions transport from surface $\mathrm{Ag}$ layer directly to subsurface Ce without any barrier and with a high exothermicity of $-3.56 \mathrm{eV}$. This result suggests that any dissociated $\mathrm{O}$ at TPB will be dragged into the unsaturated $\mathrm{Ce}^{3+}$ in the electrolyte quickly. These calculations predict that the TPB of Ag supported by Ce-terminated $\mathrm{CeO}_{2}(111)$ surface has the lowest energy barrier for $\mathrm{O}_{2}$ reduction and the dissociated $\mathrm{O}$ ions can directly transport to the $\mathrm{CeO}_{2}$ bulk without any energy barrier, implying that the TPBs are the most active sites for oxygen reduction. This result agrees well with the experimental observation that cell performance can be enhanced by increasing TPB area $[14,15]$. The stepwise reaction mechanisms are summarized in Figure 3.

\section{Calculations involving the $\mathrm{LaMnO}_{3}$ surfaces}

Shown in Figure $4 \mathrm{a}$ are the optimized geometries, O-O bonds, and their vibrational frequencies of the adsorbed oxygen species on a defective $\mathrm{LaMnO}_{3}$ (with an oxygen vacancy) Those intermediates on the defective $\mathrm{LaMnO}_{3}$ surface are energetically more favorable compared to those on a perfect surface (without oxygen vacancy). We carried out a mechanistic study for the molecular adsorption pathway at the defective $\mathrm{LaMnO}_{3}$ surface. Since we were unable to locate peroxo-like species at the La cation site, we considered only the Mn cation pathway. As depicted in Figure 4b, the first step is the formation of either superoxo-like Mn-super or peroxo-like Mn-per with exothermicities of 1.82 or $2.70 \mathrm{eV}$, respectively. As summarized in Figure $4 \mathrm{a}$, they have distinct vibrational frequencies of 1265 or $861 \mathrm{~cm}^{-1}$, respectively. Because of the further charge transfer from the surface to the adsorbate, superoxo-like Mn-super can also isomerize to the peroxo-like Mn-per intermediate after overcoming a $0.1 \mathrm{eV}$ reaction barrier of TS, leading to a lengthening of the O-O bond distance from 1.301 to $1.445 \AA$ (see Figure 1a). Then, one of the oxygen atoms of the peroxo-like Mn-per species is incorporated into the oxygen vacancy $\left(\mathrm{V}_{\mathrm{O}}{ }^{\bullet}\right)$, while breaking the $\mathrm{O}-\mathrm{O}$ bond without a well-defined transition state, producing P-V with an exothermicity of $6.99 \mathrm{eV}$. The highly exothermic process validates the good catalytic activity for oxygen dissociation on $\mathrm{LaMnO}_{3}$-based cathode materials. The monatomic oxygen species absorbed at the Mn cation diffuses to a more stable site (labeled as Products in Figure 4), which is $2.32 \mathrm{eV}$ more stable than P-V. In order to simulate SOFC conditions on the LaMnO-terminated $\mathrm{LaMnO}_{3}$ surface models, MD simulations at $800{ }^{\circ} \mathrm{C}$ were carried out. To simulate a reactant gas-phase oxygen, the distance between an $\mathrm{O}_{2}$ molecule and the surface was kept at approximately $4 \AA$ and fully optimized. The defective $\mathrm{LaMnO}_{3}$ surface was modeled to verify our minimum-energy paths (MEPs) shown in Figure $4 \mathrm{~b}$. For the MD simulations, $\Delta \mathrm{t}=2 \mathrm{fs}$ was applied and the calculations were iterated until they reached an equilibrium state. As illustrated in Figure 5, in $100 \mathrm{fs}$, the molecular adsorption of a superoxo-like species occurs. Then, one of the oxygen atoms of the adsorbed species moves toward the oxygen vacancy with energy stabilization and charge transfer, which takes place approximately in 130 fs. After the incorporation process in additional $30 \mathrm{fs}$, the adsorbed oxygen monatomic species at $\mathrm{Mn}$ cations diffuses to a more stable site. The time from adsorption to dissociation (with incorporation) was 220 fs. Even though MEP calculations (Figure 4b) determined two pathways via superoxo- and peroxo-like species without a reaction barrier, the MD simulations with our surface models suggested that the most probable reaction pathway is the formation of superoxo-like species, and then conversion to peroxo-like species with a 
small reaction barrier. Thus, we can write a possible route with oxygen vacancies on the $\mathrm{LaMnO}_{3}$-based surfaces. We summarize the most probable pathway for the oxygenreduction mechanism based on the DFT/MD modeling in Figure 3, where (g), (ad), (super), (per), and (lc) represent gas, molecular adsorption, superoxo-like species, peroxolike species, and lattice, respectively.

\section{Calculations involving LSM- and LSC-based surfaces}

To estimate the adsorption energies of oxygen species on cathode materials under SOFC operating conditions (i.e., $\mathrm{pO}_{2}=0.2 \mathrm{~atm}$ and $\mathrm{T}=873$ to $1273 \mathrm{~K}$ ), we considered the effects of temperature and pressure on gas-surface interactions by means of thermodynamiccorrection formalism. We examined the thermodynamic properties for $\mathrm{O}_{2}-\mathrm{LaMnO}_{3}$ interactions with superoxo- and peroxo-like species $\left(\mathrm{O}_{2}^{-}\right.$and $\mathrm{O}_{2}^{2-}$, respectively) at $1 / 6$ coverage. To examine the surface-orientation effect, we applied two typical adsorbed oxygen species on $\mathrm{LaMnO}_{3}(110)$ (superoxo- and peroxo-like species with the adsorption energies of $-1.76 \mathrm{eV}$ and $-2.20 \mathrm{eV}$, respectively) at $1 / 3$ coverage. As shown in Figure 7, molecularly adsorbed oxygen species may be stable at temperatures up to $\sim 1050{ }^{\circ} \mathrm{C}$ at $\mathrm{pO}_{2}$ $=0.2 \mathrm{~atm}$, depending on adsorption energies, surface orientations, and surface coverage. This information is vital to design of experiments for probing oxygen species using vibrational spectroscopy. For example, it is unlikely to observe the superoxo- and peroxolike species on the (100) surface at high temperatures; on the (110) surface, however, they are much more stable at high temperatures.

As depicted in Figure 8, for the interaction between $\mathrm{O}_{2}$ and the $\mathrm{La}_{0.5} \mathrm{Sr}_{0.5} \mathrm{MnO}_{3-\delta}$ surface, the first step is the formation of the superoxo-like super-lsm1 intermediate with an exothermicity of $1.36 \mathrm{eV}$. The diss-lsm1 species can be produced by overcoming a reaction barrier of $0.36 \mathrm{eV}$ which is much lower than that on the perfect $\mathrm{LaMnO}_{3}(\sim 0.45$ $\mathrm{eV}$ ). This means that oxygen vacancies formed by Sr doping enhance the adsorption of $\mathrm{O}_{2}$. Then, the dissociated oxygen species is incorporated into the doubly charged oxygen vacancy $\left(\mathrm{V}_{\mathrm{O}}{ }^{\bullet \bullet}\right)$ forming inc-a1 or inc-b1 with the same exothermicity of $3.90 \mathrm{eV}$. The adsorbed oxygen species on inc-a1 can further diffuse to the Mn ion on inc-b1 by overcoming a barrier of $2.09 \mathrm{eV}$. The oxygen species incorporated into the lattice can diffuse away by the hopping mechanism. In addition to molecular adsorption, oxygen molecules can directly be incorporated into oxygen vacancies via ts3-lsm1, producing incc1 with an exothermicity of $2.06 \mathrm{eV}$. The predicted rate constants for the $\mathrm{O}_{2}$ adsorption process on $\mathrm{LaMnO}_{3}(\mathbf{l m})$ and $\mathrm{La}_{0.5} \mathrm{Sr}_{0.5} \mathrm{MnO}_{3-\delta}(\mathbf{l s m})$ at $\mathrm{T}=873$ to $1273 \mathrm{~K}$ can be represented respectively by $k_{\text {ads, Im }}=1.30 \times 10^{2} \mathrm{~T}^{2.36} \mathrm{~cm}^{3} \mathrm{~s}^{-1}$ and $k_{\mathrm{ads}, \mathrm{lsm}}=3.46 \times 10^{2} \mathrm{~T}^{2.52}$ $\mathrm{cm}^{3} \mathrm{~s}^{-1}$, where the rate constants are related to the rate equation, $\mathrm{d}[\mathrm{X}]_{\text {surf }} / \mathrm{dt}=k_{\mathrm{i}}\left(\theta / \mathrm{A}_{\mathrm{s}}\right)[\mathrm{X}]_{\mathrm{g}}$, which has the units of a flux: molecule $\mathrm{cm}^{-2} \mathrm{~s}^{-1}$. In the rate equation, $\theta$ represents the fraction of available surface sites, $A_{s}$ is the surface area, and $[X]_{g}$ is the gas phase concentration of $\mathrm{O}_{2}$ in molecules $\mathrm{cm}^{-3}$. Figure 9 shows the dissociation of adsorbed oxygen species on $\mathrm{La}_{0.5} \mathrm{Sr}_{0.5} \mathrm{MnO}_{3-\delta}$ dominates over the temperature range studied, leading to the expression of $k_{\mathrm{diss}, \mathrm{lsm}}=2.15 \times 10^{12} \mathrm{exp}[-0.23 \mathrm{eV} / \mathrm{RT}] \mathrm{s}^{-1}$ at $\mathrm{P}=1 \mathrm{~atm}$ and $\mathrm{T}=873-$ $1273 \mathrm{~K}$. The predicted rate constants on the perfect $\mathrm{LaMnO}_{3}$ can be expressed: $k_{\text {diss, } I m}$ $=2.35 \times 10^{12} \exp [-0.50 \mathrm{eV} / \mathrm{RT}] \mathrm{s}^{-1}$. Our rate-constant predictions along with the mechanistic studies verify that oxygen vacancies influence $\mathrm{O}_{2}$ kinetics on SOFC cathodes. The predicted rate constants may be employed for kinetic modeling on the operation of a practical SOFC system.

Understanding surface diffusion of oxygen species on the cathode in SOFCs is very crucial to rational design of efficient cathode materials since surface diffusivity is closely 
related to the incorporation of oxygen into the lattices of cathodes or electrolytes, including diffusion to triple-phase boundaries (TPBs). Bulk diffusivities of oxygen ions in the cathode are also a significant factor because of the direct relevance to ionic conductivity. Characterizing the two diffusion phenomena under SOFC operating conditions is very important for the development of novel cathode materials; however, experimentally distinguishing the two properties is quite difficult. In this study, we predicted surface and bulk diffusivities of oxygen species on and in $\mathrm{La}_{0.5} \mathrm{Sr}_{0.5} \mathrm{MnO}_{3-\delta}$ (LSM50) and $\mathrm{La}_{0.5} \mathrm{Sr}_{0.5} \mathrm{CoO}_{3-\delta}$ (LSC50). In order to estimate surface and bulk diffusivities, we applied transition state theory (TST). The (100) surface was applied for the characterization of the surface interactions, while the (110) surface was used for bulk property calculations. As shown in Figure 10a, an adsorbed oxygen species at the Mn ions on LSM50 can migrate on the surface with a well-defined transition state. In particular, the reaction barrier for LSC50 is much lower than that for LSM50 (1.09 eV vs. $1.93 \mathrm{eV})$. Therefore, one can expect that the hopping of adsorbed oxygen species on LSC50 occurs more easily than on LSM50. As shown Figure 10b, our TST predictions of surface diffusivities clearly support that LSC-based cathode materials have a much higher mobility than LSM-based ones. Similar to the predictions of surface diffusivities of adsorbed oxygen species, we also predicted bulk diffusivities of oxygen ions in LSM50

and LSC50, showing that oxygen ion conduction through LSC-based cathode materials is much faster than that through LSM-based ones.

\section{Characterization of LSM-GDC composite cathode}

Shown in Figure 11a is a typical SEM micrograph of the dense composite layer. After performing energy dispersive X-ray (EDX) characterization on hundreds of small particles, it was found that particles with a terrace structure on their surface corresponded to LSM, while those with small dents on their surface corresponded to GDC. In this manner, the two phases were able to be differentiated. To quantify the TPB length for the various sample sets, established stereographic techniques were employed. Micrographs of the sample surfaces were taken using SEM and test lines were overlaid on the image. Using the known magnification of the micrograph, the actual total length of the test lines was determined. The number of intersections between the test lines and the LSM-GDC grain boundaries (which represents a TPB line), $\mathrm{P}_{\mathrm{L}}$, was counted. This process was repeated at different locales on the surfaces of several samples for a minimum of thirty fields of view, and an average value of the number of intersections per test line, $\left\langle\mathrm{P}_{\mathrm{L}}\right\rangle$, was calculated. The average value of the total boundary length per unit area, $\left\langle\mathrm{L}_{\mathrm{A}}\right\rangle$, is related to $\left\langle\mathrm{P}_{\mathrm{L}}\right\rangle$ through the simple expression, $\left\langle\mathrm{L}_{\mathrm{A}}\right\rangle=\pi / 2\left\langle\mathrm{P}_{\mathrm{L}}\right\rangle$. Figure $11 \mathrm{~b}$ shows a plot of inverse polarization resistance versus TPB lengths. The plot is expected to be linear but shows significant scatter, specifically from the low volume percentages of LSM samples. The main cause of this nonlinearity is due most likely to not all of the TPBs for each sample being active. For the TPB around each LSM particle to be considered active, it must contact a GDC particle that is part of a percolating cluster through to the electrolyte. The likelihood of percolation for the GDC particles becomes increasingly smaller as the volume percentage of LSM increases. It is expected that if site percolation could be taken into account and the amount of active TPB length is calculated, better linearity would be achieved. 


\section{Raman analyses}

For any of our SERS experiments, most spectra collected consisted of a fluorescence signal, likely produced by the surface metal nanoparticles themselves, which dominated over any other type of signal that might otherwise be observed. In the case of samples treated with silver, however, spectra collected from some spots displayed signals attributable to SERS effects. Such spectra, which were collected from SSC samples modified by silver colloids, are shown in Figure 12 . Typically, only about $5-10 \%$ of the points sampled from any one area treated with silver colloid generated a spectrum with a number of peaks comparable to the spectrum in Figure 12. The relative scarcity of these spots supports the theory that the effect only occurs in certain "hot spots" where the metal nanostructures happen to be in a favorable configuration. While there are many peaks in these spectra that require identification, the spectra show evidence of adsorbed molecular oxygen (near $1530 \mathrm{~cm}^{-1}$ ) and adsorbed reduced oxygen (800-1100 $\mathrm{cm}^{-1}$ range) [16]. The ability to observe how these peaks change under SOFC operating conditions would help elucidate the oxygen reduction process. It should be noted that these spectra only came from silver-treated samples, however. The samples treated with gold nanoparticles did not produce a surface-specific SERS signal in any one spot measured, possibly due to the fact that $514 \mathrm{~nm}$, our Raman spectrometer's wavelength, is below the absorption maximum of the gold nanoparticles.

Samples treated by sputtering showed slightly different behavior. Figure 13 displays spectra collected from LSCF and LSM sample surfaces with discontinuous silver films. While the spectra show indication of signal enhancement, the potential reduced oxygen species features are typically not as apparent as with some of the spectra with signal enhanced by colloids. That being said, a larger percentage $(\sim 10-20 \%)$ of the sample points collected from the sputtered samples show signs of SERS than those collected from colloidal samples. The enhanced adsorbed molecular oxygen peak is usually present with the sputtered samples, as well. As can be seen above, the same features in general can be found in spectra from LSCF and LSM samples, but with slight shifts and varying degrees of enhancement, suggesting that the signals originate from the surface of the cathode samples rather than the enhancing nanoparticles themselves.

We have also analyzed both sputter-treated cathode samples under atmospheres that contain only oxygen and inert gas (Ar) to examine the effects on the spectra and isolate oxygen peaks. Figures 14 and 15 shows these effects. As can be observed in the Figure 14 (the sputtered samples), some peaks in the $800-1200 \mathrm{~cm}^{-1}$ range change upon exposure, supporting the claim that at least some of these features correspond to reduced oxygen species. In addition, the features in Figure 14, which may correspond to disordered carbon and graphite [17], disappear in the spectrum from the oxygen-exposed sample. This may happen due to oxidation of carbon. The colloidal samples examined in this manner (Figure 15) had a more rigorous cleaning treatment applied, and as a result, many of the peaks found in spectrum A disappear in Spectra B and C, and the underlying broad carbon signals, centered near at $1340 \mathrm{~cm}^{-1}$ and $1570 \mathrm{~cm}^{-1}$, also vanish. Figure $1 \mathrm{~B}$ and $1 \mathrm{C}$, in comparison to SERS spectra we have obtained in the past, contain many less peaks, as well as peaks that are easier to identify. For example, the peak at $1535 \mathrm{~cm}^{-1}$ in Spectrum $\mathrm{B}$ can be assigned to adsorbed neutral oxygen species. Different peaks enhanced in Spectrum $\mathrm{C}$ were enhanced. The assignment of these peaks is less clear, although they promisingly fall within the range of reduced oxygen species, especially the peak near 990 $\mathrm{cm}^{-1}$. 
Some of our other work has involved the use of Raman spectroscopy to characterize the degradation of LSCF under polarization. One possible reason for degradation from polarization is the transport of strontium from the lattice to the surface, forming a layer of SrO. This layer would reduce the catalytic activity on the surface of the material, and increase the ohmic resistance of the cathode due to the poor electronic conductivity of SrO. Figure 16 (a) shows spectra collected from a $1 \mu \mathrm{m}$ LSCF film on a gadolinium-doped ceria (GDC) substrate before and after exposure to $0.8 \mathrm{~V} \mathrm{DC}$ polarization at $700^{\circ} \mathrm{C}$. A shift in the peaks can be observed, as well as an increase in the ratio of the right peak to the left. According to LSCF powders of various stoichiometries that were also measured by Raman spectroscopy (Fig. 16b), this change possibly corresponds to an increase in the $\mathrm{La} / \mathrm{Sr}$ ratio in the lattice, suggesting the migration of $\mathrm{Sr}$ out of the lattice that may form its own oxide on the surface.(09)

Raman spectroscopy was explored as a method for characterizing cathode thin films. As can be seen in Figure 17, which shows spectra collected from various LSCF films deposited on GDC substates, Raman can detect signal from the band near $640 \mathrm{~cm}^{-1}$, which corresponds to LSCF, for films thinner than $100 \mathrm{~nm}$. In addition, the signal for these thin films is much more apparent for the same amount of collection than that of a spectrum for a 1-mm thick pellet of LSCF, which is also shown in Figure 17. This may be due to a uniform orientation of the film crystals that are deposited on the substrate. The grain size for these crystals may also be large. 


\section{CONCLUSIONS}

Under the support of this project, we have made progress in the elucidation of the mechanisms of oxygen reduction of perovkite-type cathode materials for SOFCs using quantum chemical calculations. We established computational framework for predicting properties such as oxygen diffusivity and reaction rate constants for adsorption, incorporation, and TPB reactions. We have successfully formulated such predictions for LSM-based and LSCF-based cathode materials.

We have also further developed Raman spectroscopy as well as SERS as a characterization tool for SOFC cathode materials. We utilized SERS to find evidence of pertinent oxygen species on the cathode surface under atmospheric conditions. Raman spectroscopy was also shown to have the capability to detect changes in cathode chemistry that occur as a result of fuel cell operating conditions. We also showed that it can characterize cathode films with thicknesses $<100 \mathrm{~nm}$ in this manner.

Much work on the subject of unraveling oxygen reduction for SOFC cathodes remains to be done, however. For example, Raman spectroscopy could be used in conjunction with electrochemical techniques to further validate the findings of the quantum chemical calculations. 
Figures
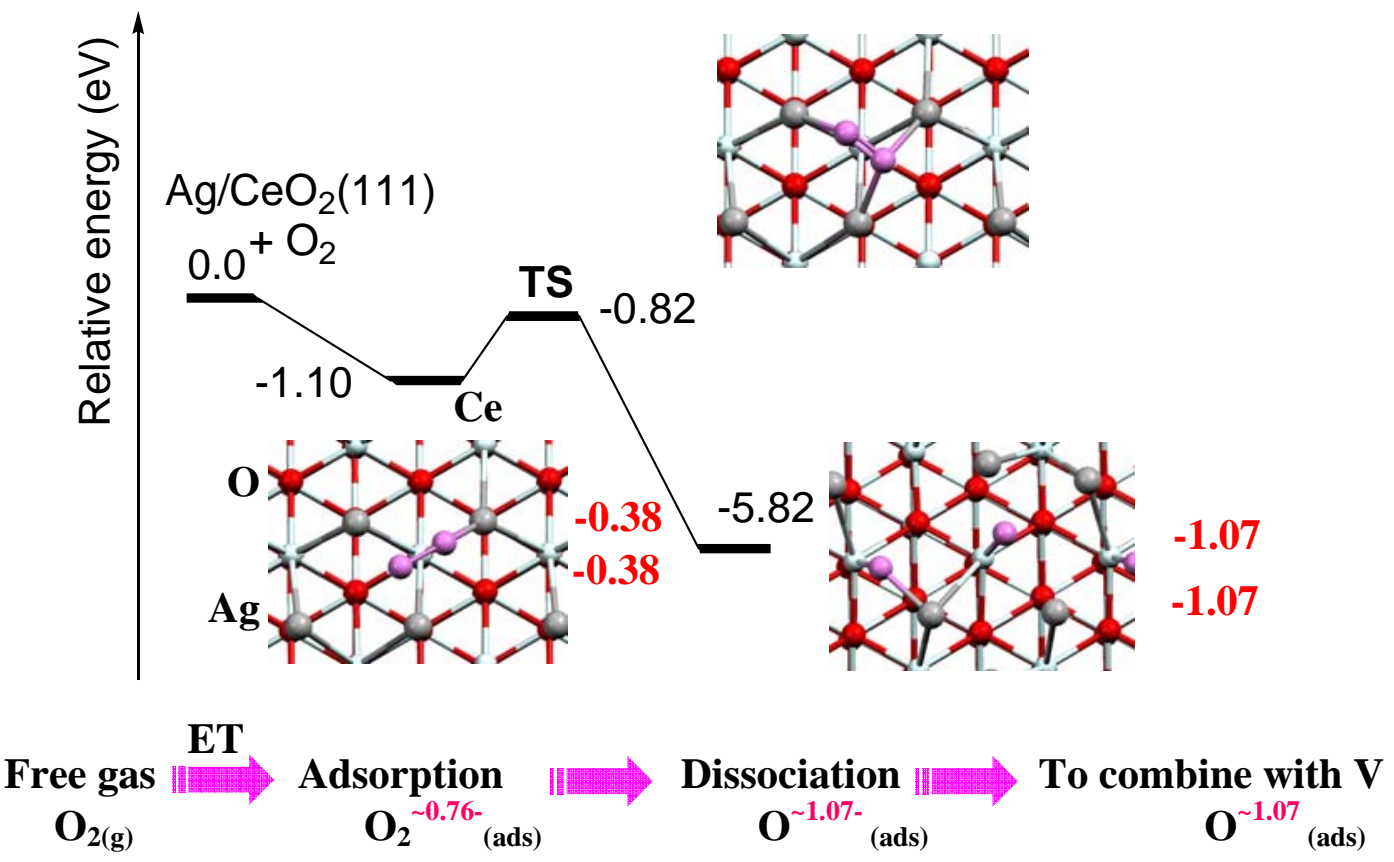

Figure 1: PES and geometrical structures of $\mathrm{O}_{2}(\mathrm{a}) \rightarrow 2 \mathrm{O}$ (a) dissociation processes on $\mathrm{Ag}$ covered Ce-terminated $\mathrm{CeO}_{2}(111)$ surface. The black numbers are the relative energies referenced to $\mathrm{O}_{2}(\mathrm{~g}) /$ surfaces $(=0)$. The red numbers are the Bader charges of the adsorbed $\mathrm{O}$.
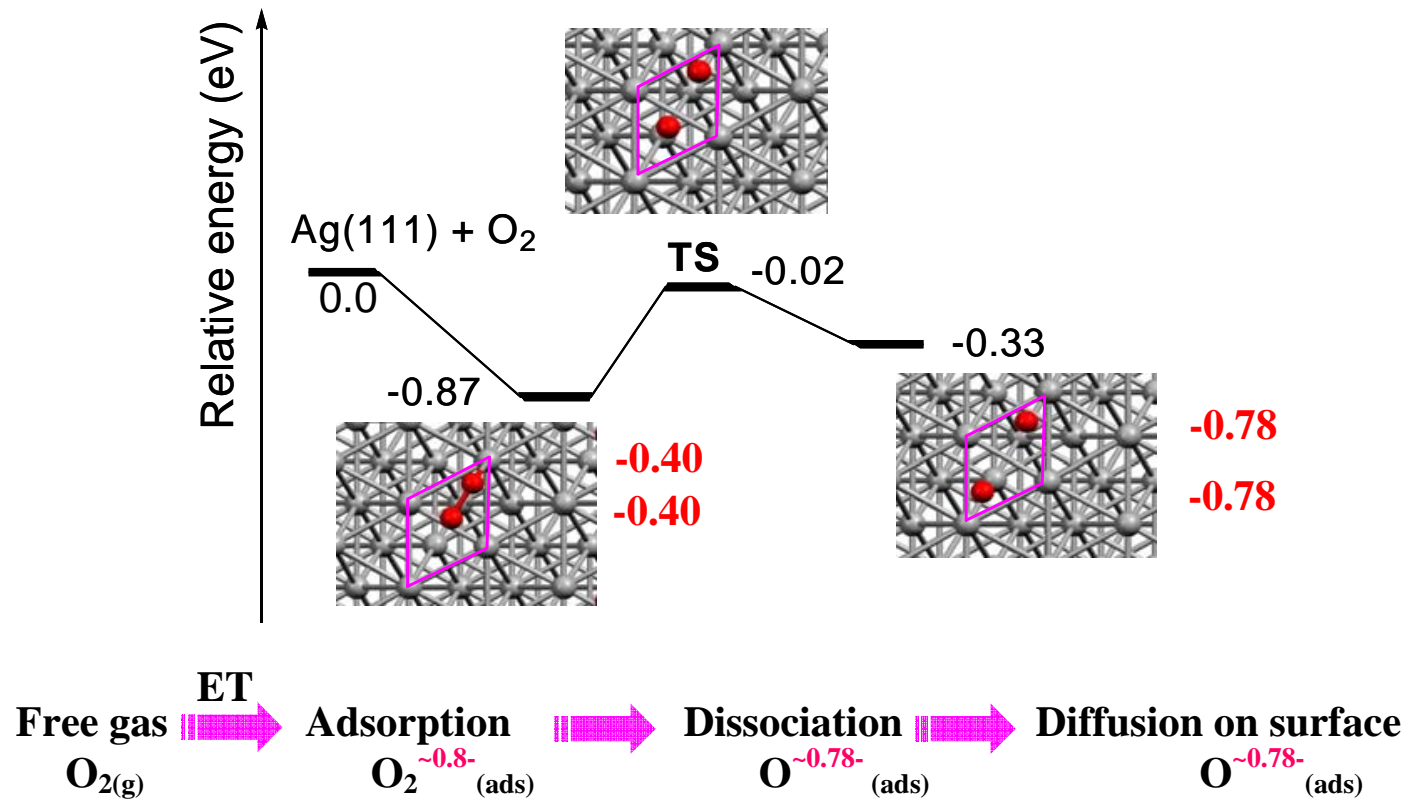

Figure 2: PES and geometrical structures of $\mathrm{O}_{2}$ (a) $\rightarrow 2 \mathrm{O}$ (a) dissociation processes on $\mathrm{Ag}(111)$ surface. The black numbers are the relative energies referenced to $\mathrm{O}_{2}(\mathrm{~g}) /$ surfaces $(=0)$. The red numbers are the Bader charges of the adsorbed $\mathrm{O}$. 


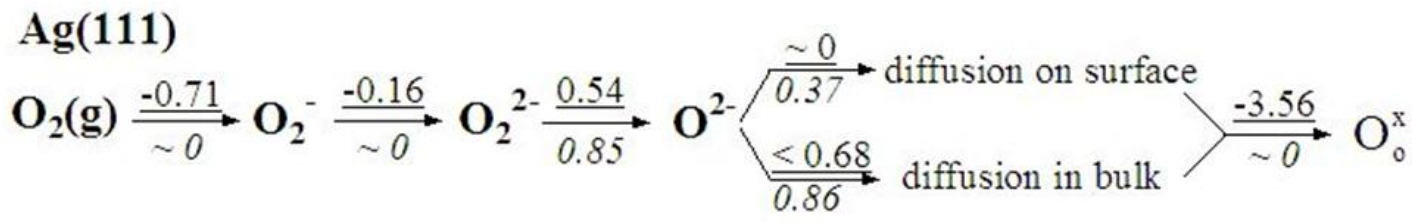

$\operatorname{Ag}(110)$

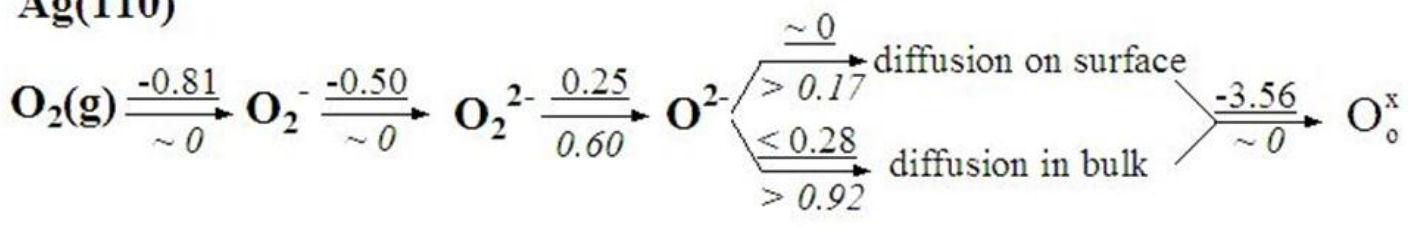

\section{$\mathrm{Ag} / \mathrm{CeO}_{2}$}

$\mathbf{O}_{2}(\mathbf{g}) \underset{\sim 0}{\stackrel{-(1.41}{\longrightarrow}} \mathbf{O}_{2} \underset{\sim 0}{-\underset{\sim 0.21}{\longrightarrow}} \mathbf{O}_{2}{ }_{2}^{2-} \underset{0.43}{\stackrel{-0.44}{\longrightarrow}} \mathbf{O}^{2-} \frac{\stackrel{\sim 0}{\longrightarrow}}{0.60}$ diffusion on surface

$\Delta \mathrm{H}: \#(\mathrm{eV}) ; \mathrm{Ea}: \#(\mathrm{eV})$

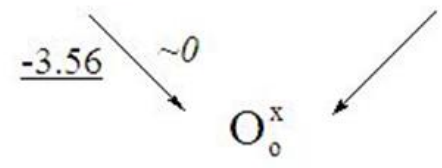

Figure 3. The stepwise reaction mechanisms of oxygen reduction processes at $\mathrm{Ag}(111)$ and $\mathrm{Ag}(110)$ surfaces and at $\mathrm{TPB}, \mathrm{CeO}_{2}(111)$-supported silver surface and Ce-terminated $\mathrm{CeO}_{2}(111)$ surface The underlined numbers represent the heat of reaction, $\Delta \mathrm{H}$, in $\mathrm{eV}$ whereas the italic numbers represent the reaction barrier, $\mathrm{Ea}$, in $\mathrm{eV}$.

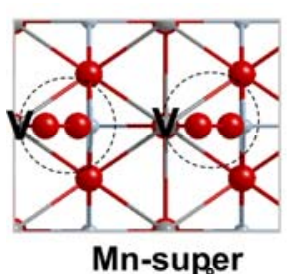

Mn-super

$\mathrm{r}_{\mathrm{OO}}=1.301 \mathrm{~A}$

$v_{\mathrm{OO}}=1265 \mathrm{~cm}^{-1}$

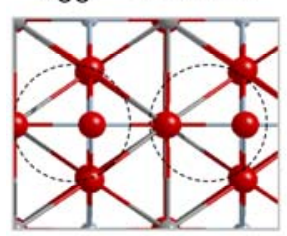

P.V

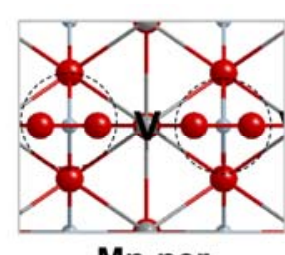

Mn-per. $\mathrm{r}_{\mathrm{OO}}=1.445 \AA$
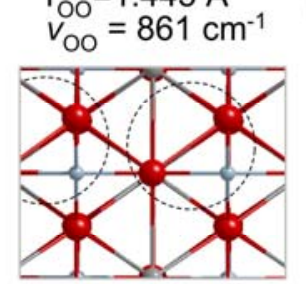

Products

(a)

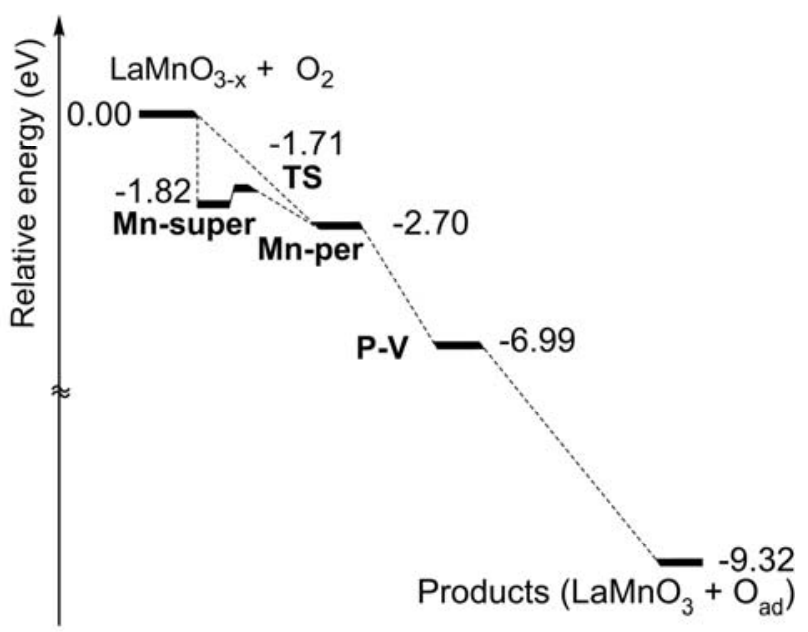

(b)

Figure 4. (a) Geometrical information of adsorbed oxygen species on a defective $\mathrm{LaMnO}_{3}$ surface. $\mathbf{V}$ and dashed circles denote an oxygen vacancy and adsorbed oxygen species on the surface, respectively. (b) Potential energy profiles at $0 \mathrm{~K}$ for the oxygen reduction reaction on a defective $\mathrm{LaMnO}_{3}$ surface. 


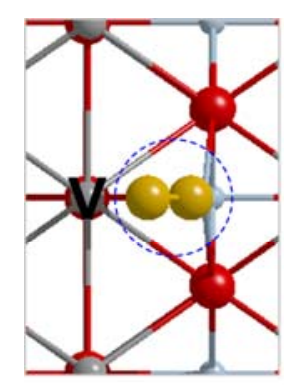

I. $0 \mathrm{fs}$

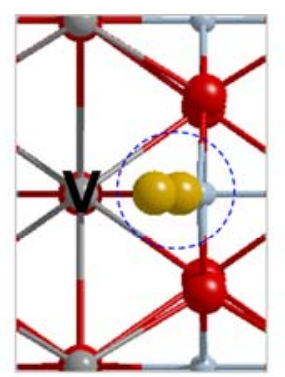

II. 100 fs

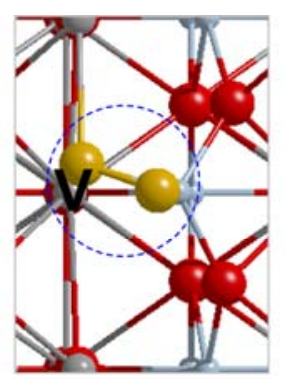

III. $230 \mathrm{fs}$

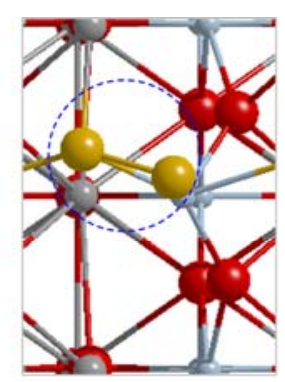

IV. $260 \mathrm{fs}$

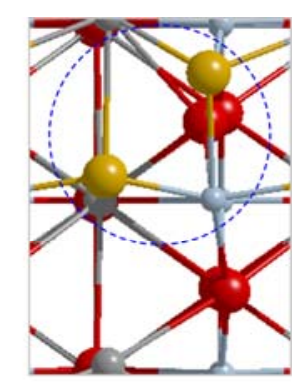

V. $320 \mathrm{fs}$

(a)

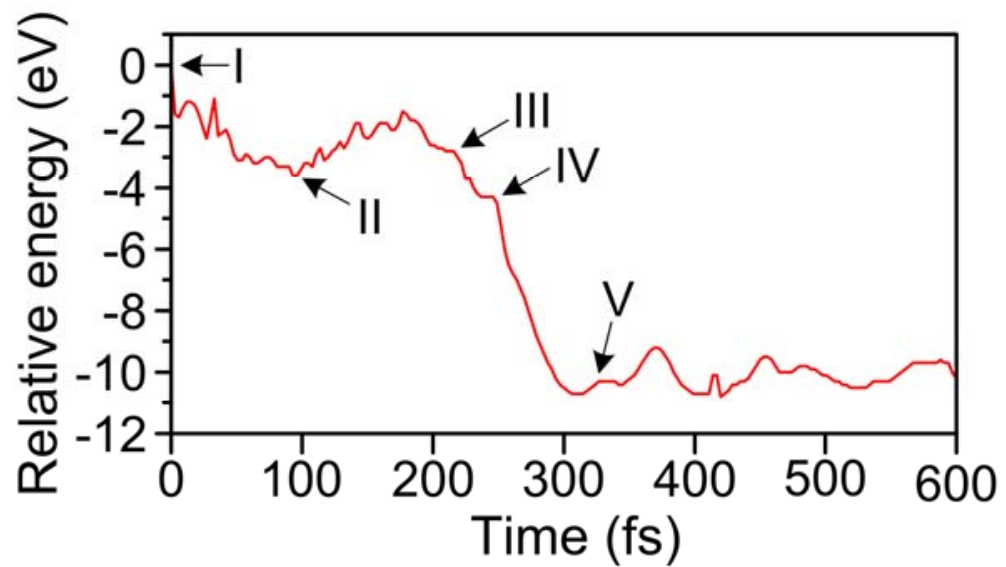

(b)

Figure 5. Molecular dynamics (MD) results simulated at $800^{\circ} \mathrm{C}$. V and dashed circles denote an oxygen vacancy and adsorbed oxygen species on the surface, respectively.

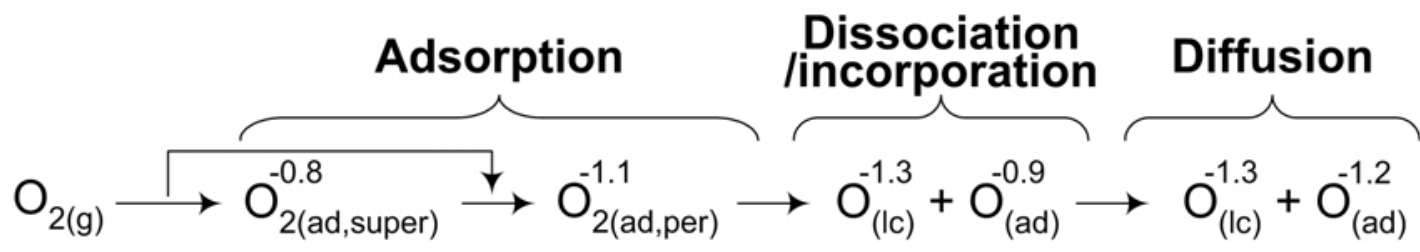

Figure 6. A stepwise reaction mechanism of oxygen reduction on a defective LMO surface. 


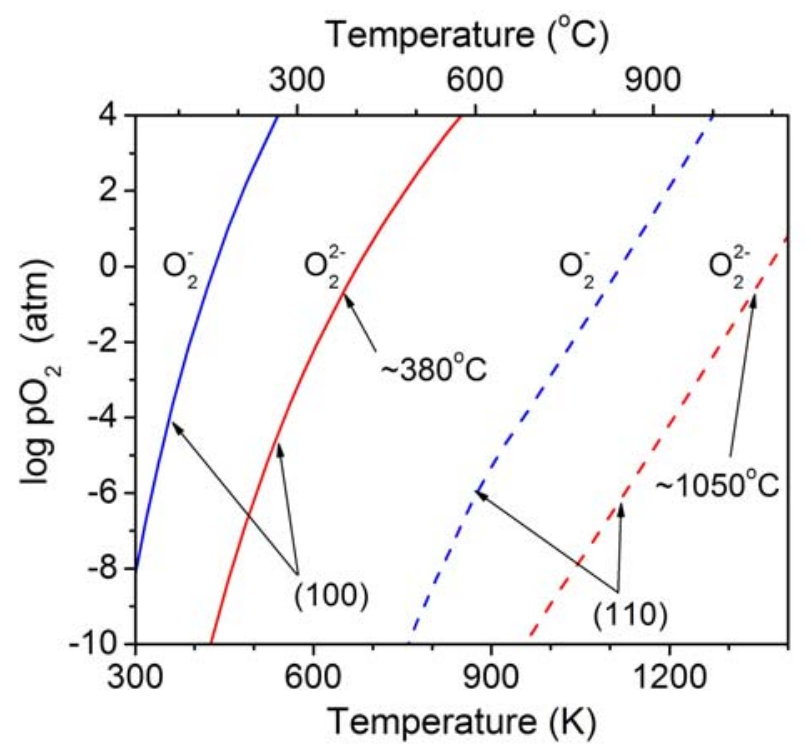

Figure 7. A typical phase diagram for the interactions between $\mathrm{O}_{2}$ and $\mathrm{LaMnO}_{3}(100)$ and (110) surfaces at $1 / 6$ and $1 / 3$ coverage, respectively.

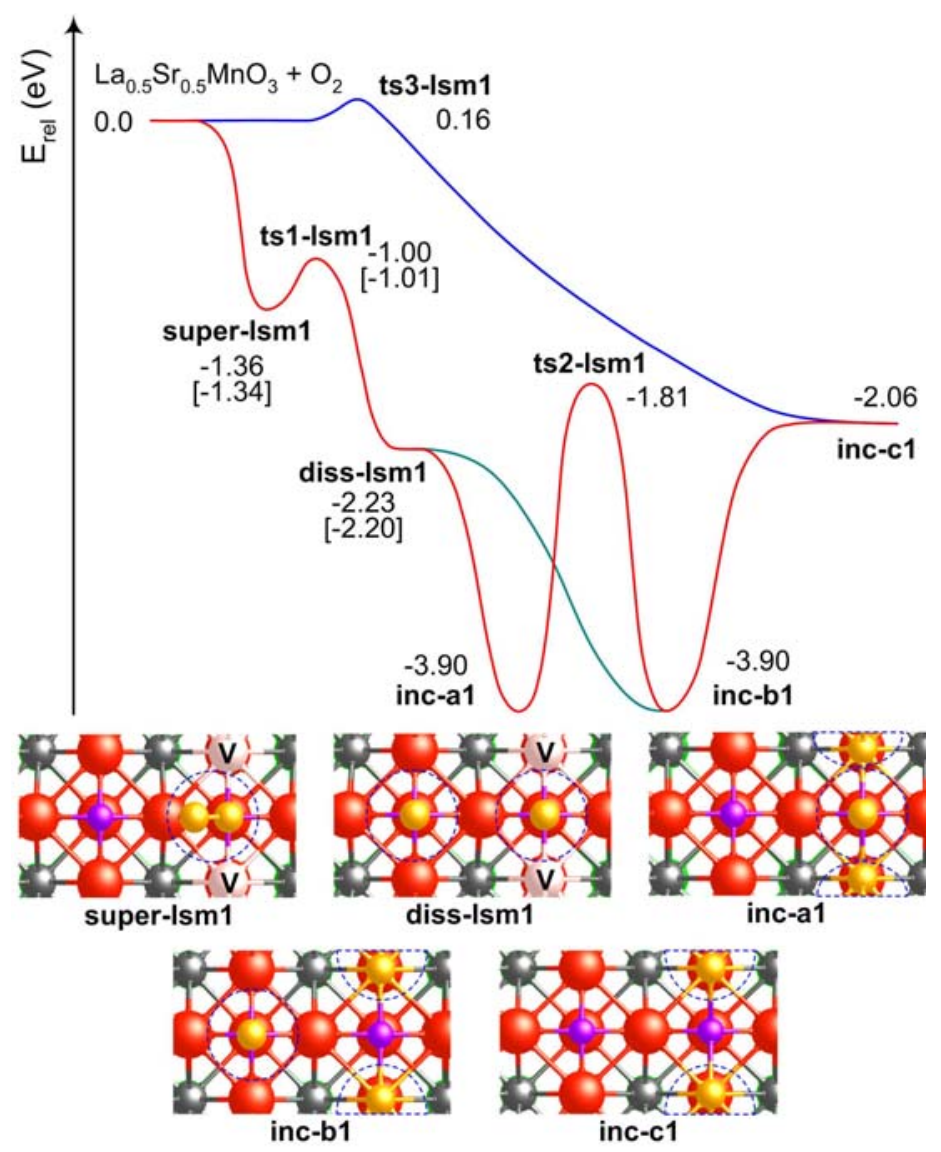

Figure 8. Potential energy profiles and top views of an intermediate state and products for $\mathrm{O}_{2}$ $\mathrm{La}_{0.5} \mathrm{Sr}_{0.5} \mathrm{MnO}_{3-\delta}$ interactions. $\mathbf{V}$ denotes an oxygen vacancy. Dashed circles and $\mathbf{V}$ represent adsorbed oxygen species and an oxygen vacancy on the surface, respectively. Relative energies in brackets are ZPE-corrected. 


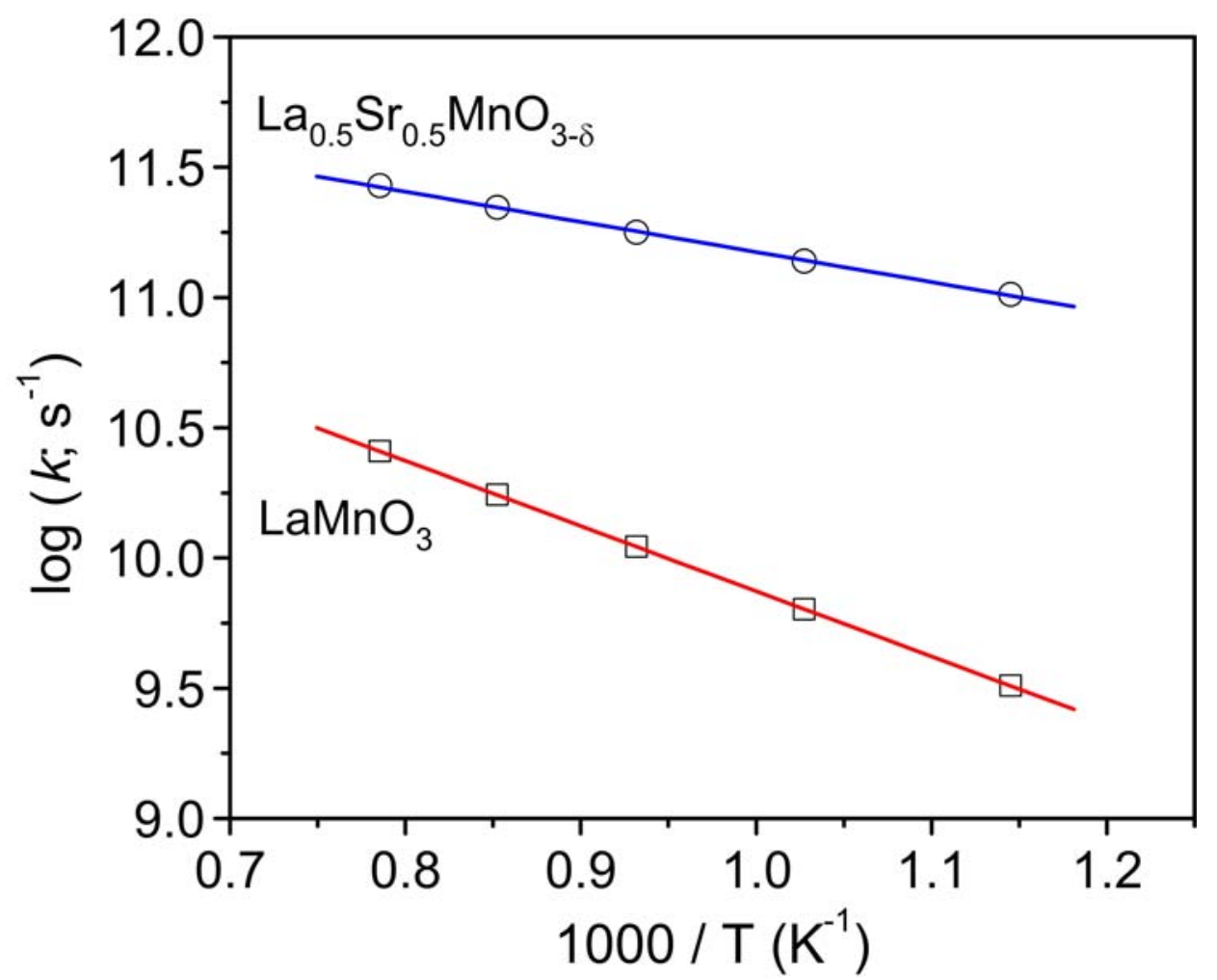

Figure 9. Comparison of predicted rate constants for the dissociation process of $\mathrm{O}_{2}$ on the perfect $\mathrm{LaMnO}_{3}$ and $\mathrm{La}_{0.5} \mathrm{Sr}_{0.5} \mathrm{MnO}_{3-\delta}$. Solid lines are fitted using the linear-squared method.

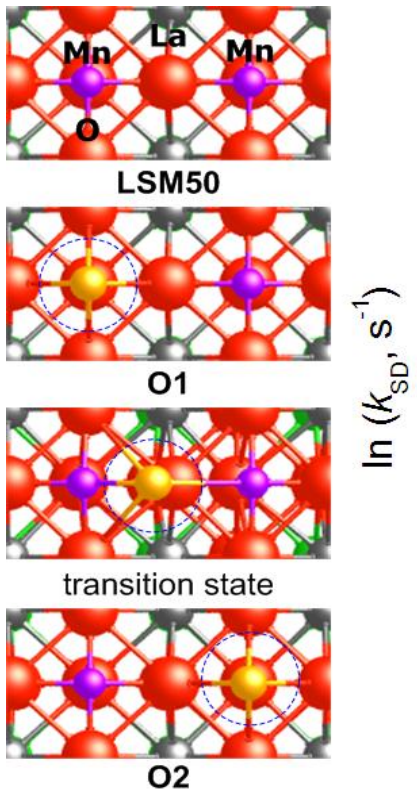

(a)

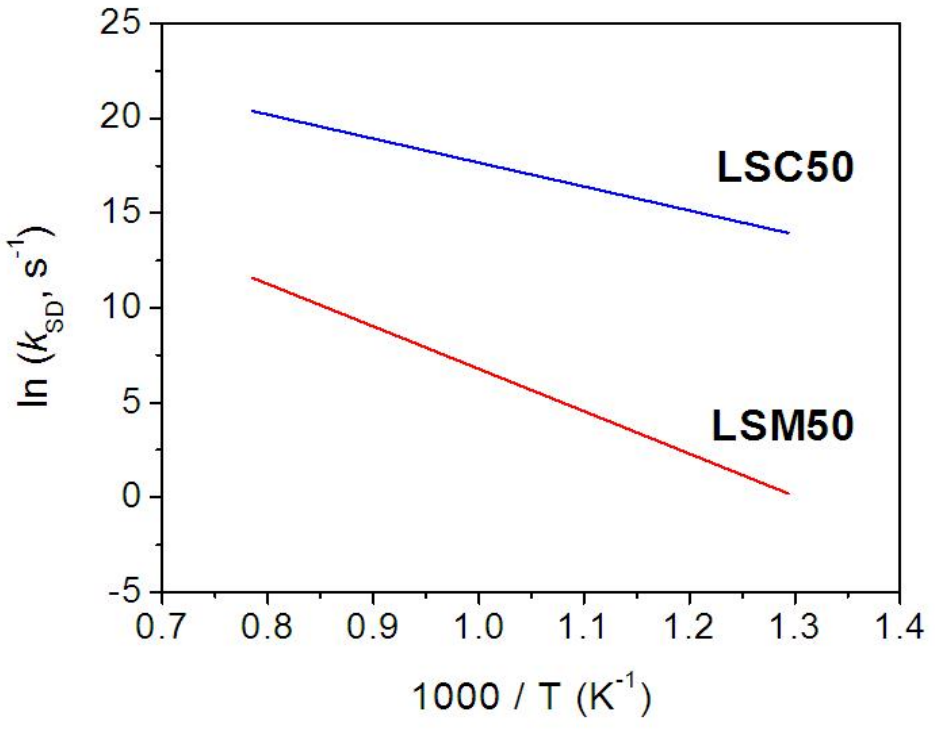

(b)

Figure 10. (a) Top views of the LSM50(100) surface model and stable states (O1 and O2) and a transition state of adsorbed oxygen species on LSM50. (b) Predicted surface diffusivities $\left(k_{\mathrm{SD}}\right)$ of adsorbed oxygen species on LSM50 and LSC50. 


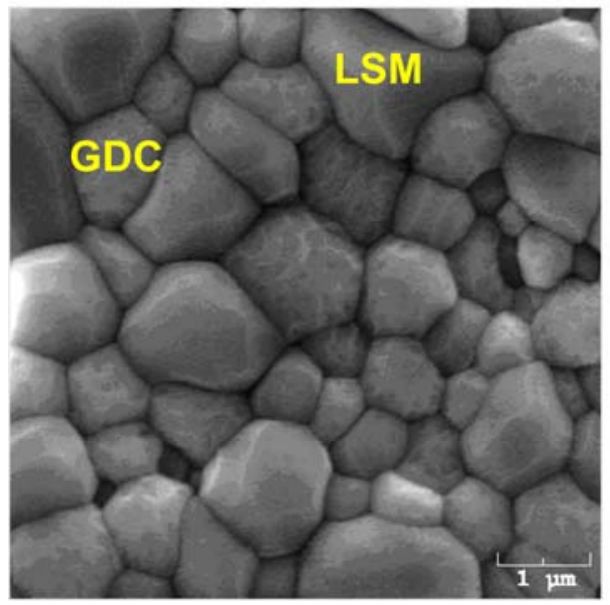

(a)

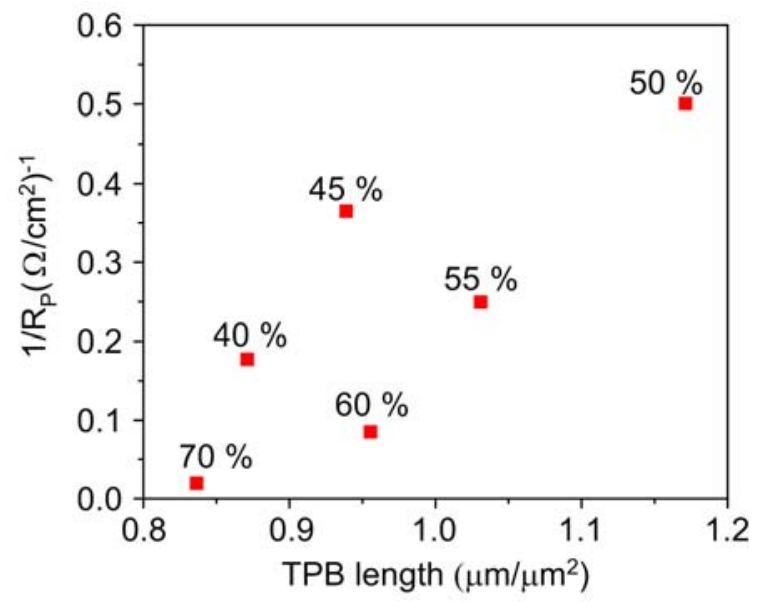

(b)

Figure 11. (a) SEM image of a dense LSM-GDC composite cathode fabricated by a co-pressing and co-sintering method. (b) Inverse polarization resistance versus TPB length. The values next to the data points are LSM volume percentages for the samples.
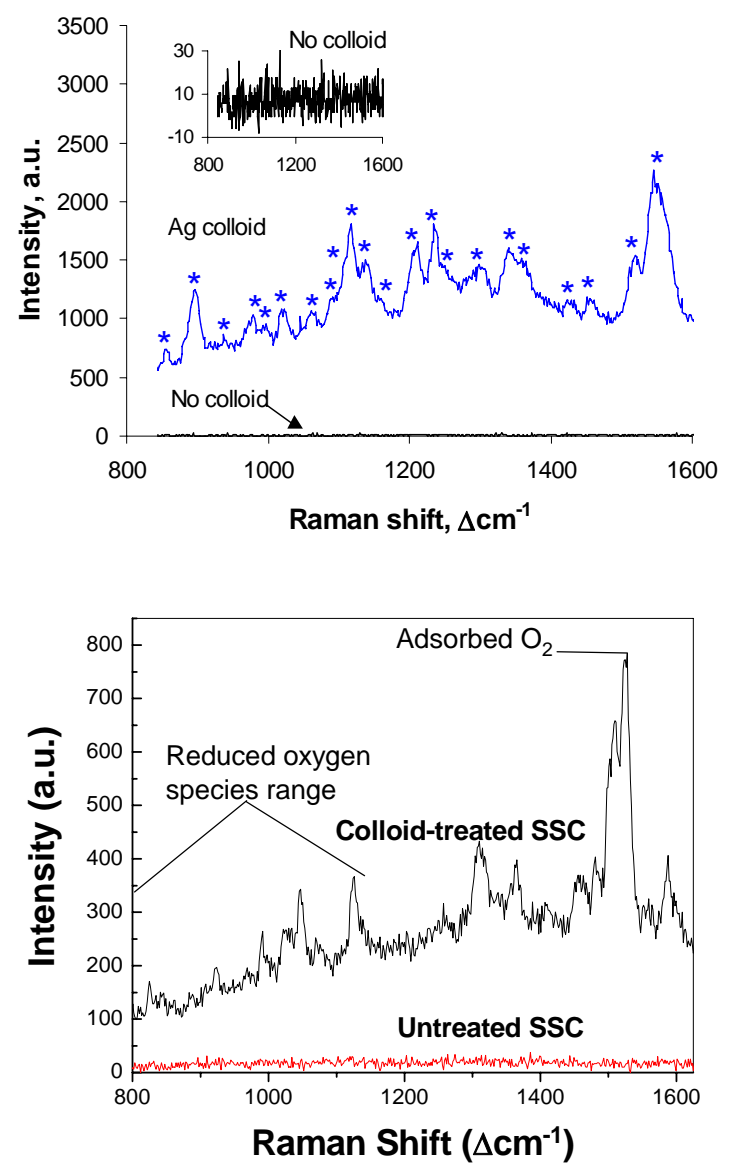

Figure 12: Raman spectra collected from various SSC samples modified with $20 \mathrm{~nm}$ Ag colloid in air. Different SERS hotspots may enhance different species signals and thus different peaks. 


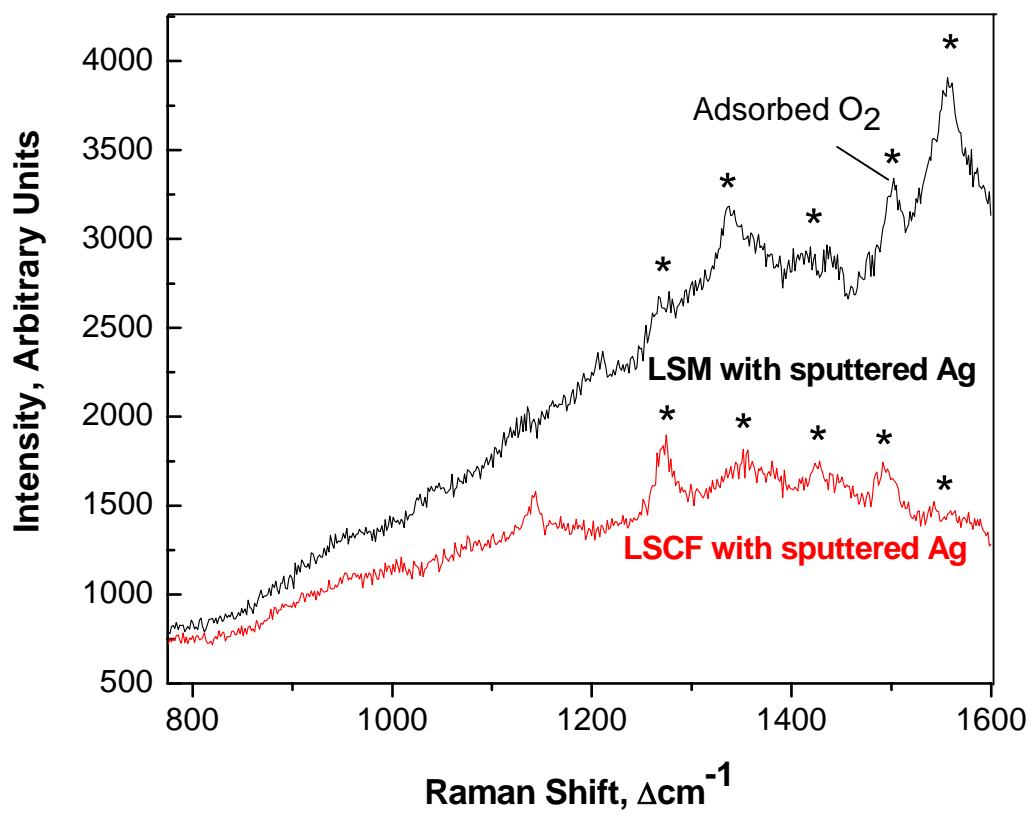

Figure 13. Raman spectra for LSM and LSCF pellets in air after DC sputtering of Ag for 10 seconds.

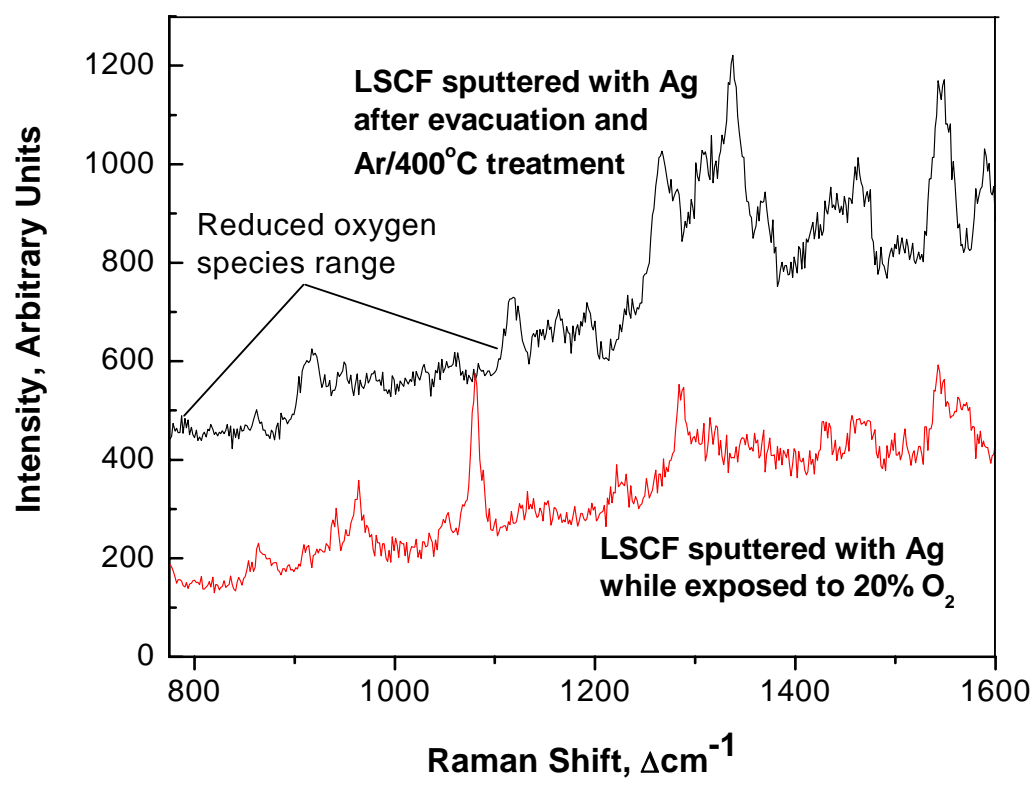

Figure 14: Raman spectra collected at room temperature from LSCF with sputtered Ag under various controlled atmosphere conditions (argon and oxygen gases). 


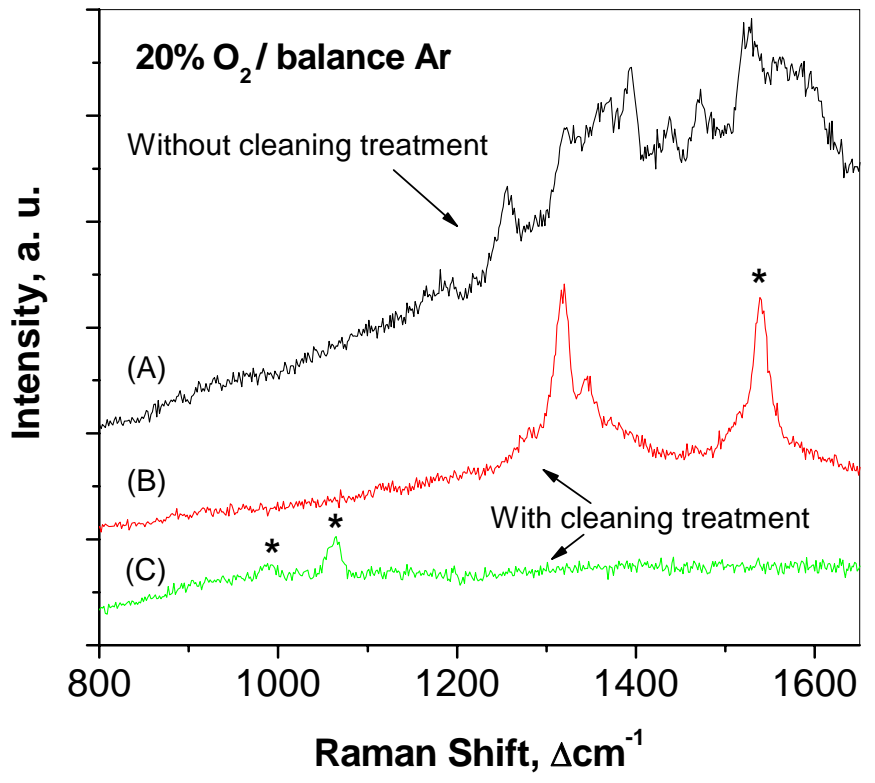

Figure 15. Raman spectra obtained from Ag colloid-treated LSCF surface at room temperature under controlled atmosphere. 


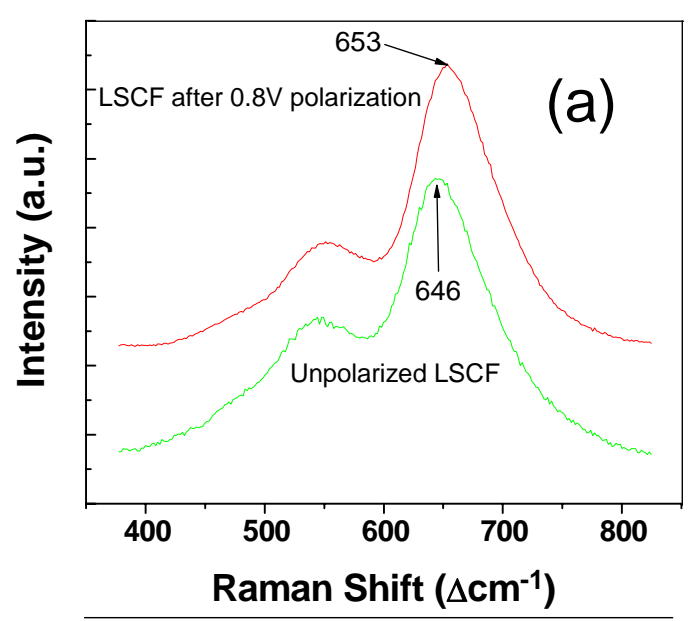

(b)

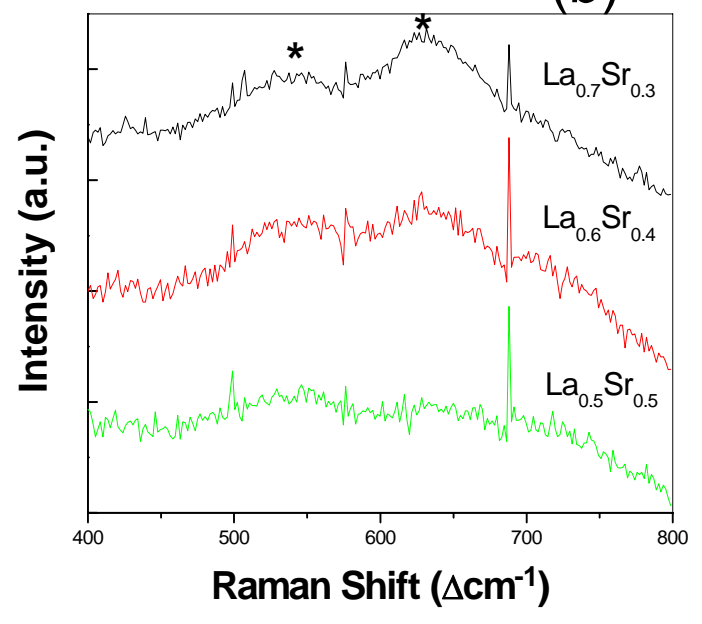

Figure 16: (a) Raman spectrum collected from surface of LSCF film on GDC pellet in air before and after application of $0.8 \mathrm{~V}$ DC polarization. A shift in the wavenumbers of the peaks can be seen, and the ratio of the larger peak to the smaller one also increases..(b) Raman spectra collected from powders of different $\mathrm{LSCF}\left(\mathrm{La}_{1-\mathrm{x}} \mathrm{Sr}_{\mathrm{x}} \mathrm{Co}_{0.2} \mathrm{Fe}_{0.8} \mathrm{O}_{3-\delta}\right)$ compositions. The two marked peaks vary with change in $\mathrm{La} / \mathrm{Sr}$ ratio. 


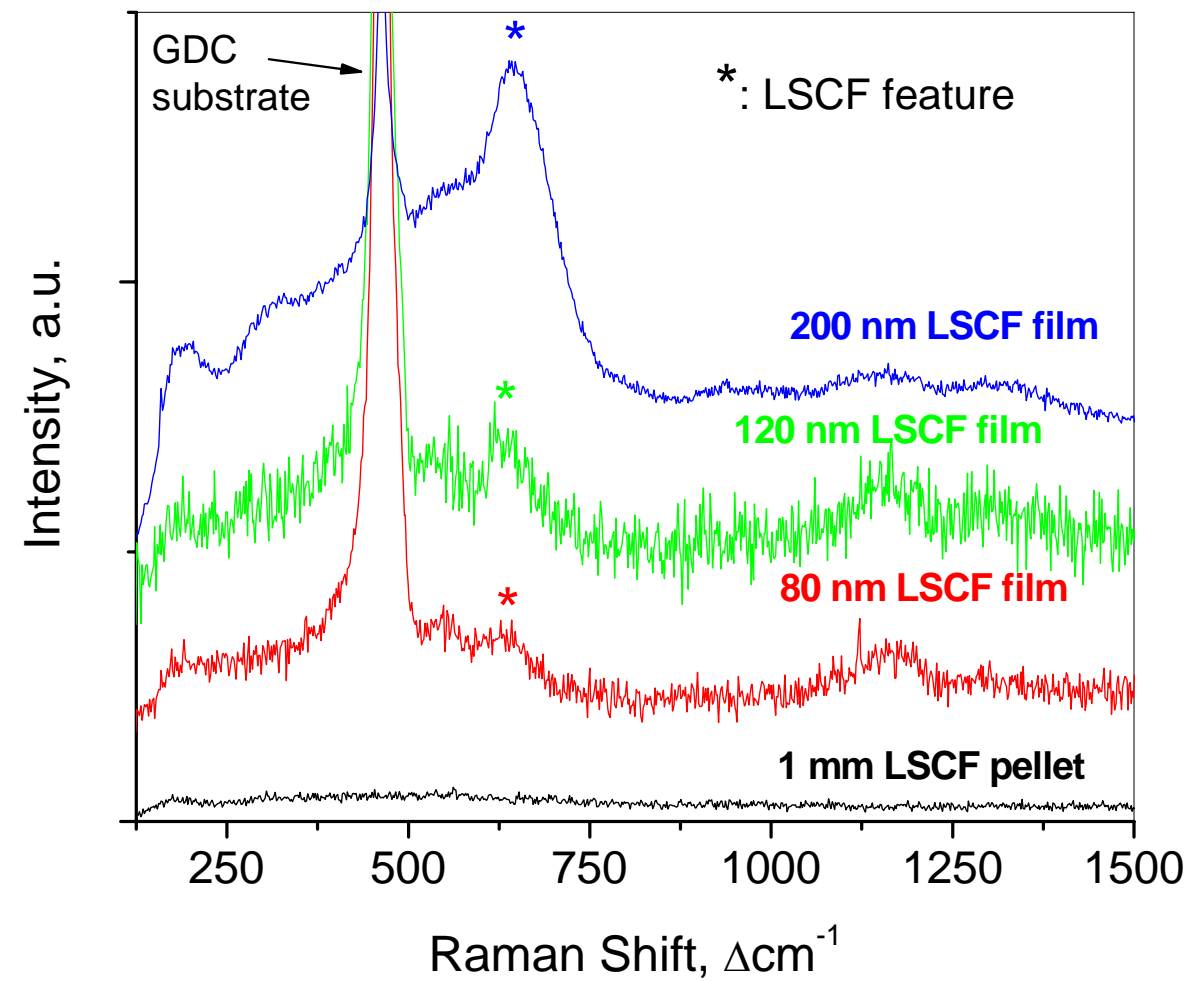

Figure 17. Raman spectra collected in air from sputter-deposited LSCF films on GDC substrates of various thicknesses, as well as a cylindrical pellet of LSCF. 


\section{References}

1. M. Liu and J. Winnick, Solid State Ionics, 118 (1999) 11-21.

2. J. Fleig, Annual Review of Materials Research 33 (2003) 361.

3. P.E. Blöchl, Physical Review B 50 (1994) 17953.

4. G. Kresse, D. Joubert, Physical Review B 59 (1999) 1758.

5. J.P. Perdew, J.A. Chevary, S.H. Vosko, K.A. Jackson, M.R. Pederson, D.J. Singh, C. Fiolhais, Physical Review B 46 (1992) 6671.

6. H.J. Monkhorst, J.D. Pack, Physical Review B 13 (1976) 5188.

7. Y.M. Choi, D. S. Mebane, M. C. Lin, M. Liu, "Oxygen Reduction on $\mathrm{LaMnO}_{3}$-based Cathode Materials in Solid Oxide Fuel Cells," Chemistry of Materials, 19, 1690, 2007.

8. Y.M. Choi, M. C. Lin, M. Liu, "Computational Study on the Catalytic Mechanism toward Oxygen Reduction on $\mathrm{La}_{0.5} \mathrm{Sr}_{0.5} \mathrm{MnO}_{3}(110)$ in Solid Oxide Fuel Cells," Angewandte Chemie, International Edition, 46, 7214-7219, 2007

9. Y.M. Choi, M.E. Lynch, M.C. Lin, M. Liu, Journal of Physical Chemistry C, 113 (2009) 7290-7297

10. Y.M. Choi, M.C. Lin, M. Liu, Journal of Power Sources 195 (2010) 1441-1445.

11. V.V. Pushkarev, V.I. Kovalchuk, J.L. d'Itri, Journal of Physical Chemistry B 108 (2004) 5341.

12. R.D. Jones, D.A. Summerville, F. Basolo, Chemical Reviews 79 (1979) 13.

13. R.F.W. Bader, Atoms in Molecules - A Quantum Theory, Oxford, Oxford University Press, 1990.

14. T. Horita, K. Yamajia, N. Sakaia, Y. Xionga, T. Katoa, H. Yokokawa, T. Kawada, Journal of Power Sources 106 (2002) 224.

15. R. Radhakrishnan, A.V. Virkar, S.C. Singhalb, Journal of The Electrochemical Society 152 (2005) A927.

16. Y.M. Choi, H. Abernathy, H.-T. Chen, M.C. Lin, M. Liu, "Characterization of $\mathrm{O}_{2-}$ $\mathrm{CeO}_{2}$ Interactions Using in Situ Raman Spectroscopy and First-Principle Calculations," ChemPhysChem, 7, 1957-1963, 2006.

17. M. Pomfret, J.C. Owrutsky, R.A. Walker, "In Situ Studies of Fuel Oxidation in Solid Oxide Fuel Cells," Analytical Chemistry, 79, 2367-2372, 2007. 